\title{
1 Large-scale Genetic Characterization of a Model 2 Sulfate-Reducing Bacterium
}

5 Valentine V. Trotter ${ }^{1,7}$, Maxim Shatsky ${ }^{1}$, Morgan N. Price ${ }^{1}$, Thomas R. Juba ${ }^{2}$, Grant M. Zane ${ }^{2}$, Kara B. De 6 León ${ }^{2,6}$, Erica L. Majumder ${ }^{2,5}$, Qin Gui ${ }^{2}$, Rida Ali ${ }^{1}$, Kelly M. Wetmore ${ }^{1}$, Jennifer V. Kuehl ${ }^{1}$, Adam P. 7 Arkin ${ }^{1,3}$, Judy D. Wall ${ }^{2}$, Adam M. Deutschbauer ${ }^{1,4}$, John-Marc Chandonia ${ }^{1,7}$ and Gareth P. Butland ${ }^{1}$

$9{ }^{1}$ Environmental Genomics and Systems Biology Division, Lawrence Berkeley National Laboratory, 10 Berkeley, CA, USA

$11{ }^{2}$ Department of Biochemistry, University of Missouri, Columbia, MO, USA

$12{ }^{3}$ Department of Bioengineering, University of California, Berkeley, CA, USA

$13{ }^{4}$ Department of Plant and Microbial Biology, University of California, Berkeley, CA, USA

$14{ }^{5}$ Current address: Department of Bacteriology, University of Wisconsin-Madison, Madison, WI, USA

$15{ }^{6}$ Current address: Department of Microbiology and Plant Biology, University of Oklahoma, Norman, OK, 16 USA

$17{ }^{7}$ To whom correspondence should be addressed:

18 VVT: vvtrotter@lbl.gov

19 JMC: jmchandonia@lbl.gov 


\section{ABSTRACT}

27 Sulfate-reducing bacteria (SRB) are obligate anaerobes that can couple their growth to the reduction of

28 sulfate. Despite the importance of SRB to global nutrient cycles and their damage to the petroleum industry,

29 our molecular understanding of their physiology remains limited. To systematically provide new insights

30 into SRB biology, we generated a randomly barcoded transposon mutant library in the model SRB

31 Desulfovibrio vulgaris Hildenborough $(\mathrm{DvH})$ and used this genome-wide resource to assay the importance

32 of its genes under a range of metabolic and stress conditions. In addition to defining the essential gene set

33 of $\mathrm{DvH}$, we identified a conditional phenotype for 1,137 non-essential genes. Through examination of these

34 conditional phenotypes, we were able to make a number of novel insights into our molecular understanding

35 of DvH, including how this bacterium synthesizes vitamins. For example, we identified DVU0867 as an

36 atypical L-aspartate decarboxylase required for the synthesis of pantothenic acid, provided the first

37 experimental evidence that biotin synthesis in DvH occurs via a specialized acyl carrier protein and without

38 methyl esters, and demonstrated that the uncharacterized dehydrogenase DVU0826:DVU0827 is necessary

39 for the synthesis of pyridoxal phosphate. In addition, we used the mutant fitness data to identify genes

40 involved in the assimilation of diverse nitrogen sources, and gained insights into the mechanism of

41 inhibition of chlorate and molybdate. Our large-scale fitness dataset and RB-TnSeq mutant library are

42 community-wide resources that can be used to generate further testable hypotheses into the gene functions

43 of this environmentally and industrially important group of bacteria.

44

45

46

47

48

49

50

51

52

53 
Sulfate-reducing bacteria (SRB) are present in diverse anoxic environments including the deep ocean, where they are responsible for a considerable fraction of carbon mineralization (Muyzer and Stams, 2008), and in the human gut, where they produce hydrogen sulfide (Kushkevych et al., 2020). Utilization of SRB has been extensively explored for bioremediation (eg. heavy metals, radionuclides) by metabolismdependent mechanisms and/or bioaccumulation (Joo et al., 2015; Mikheenko et al., 2008; Rückert, 2016;

63 Yong et al., 2002). In the oil and gas industry, the activity of SRB leads to undesirable effects including 64 souring of oil and corrosion of pipelines (Kip and van Veen, 2015; Thrasher and Vance, 2005). Given their 65 importance, it is imperative that we develop a detailed gene-level characterization of SRB to understand and control their activities in diverse environments.

Much of our molecular understanding of SRB is derived from studies in the model Desulfovibrio vulgaris Hildenborough (DvH), which was the first SRB to have its genome sequenced (Heidelberg et al., 2004). DvH has an established genetic toolkit including a markerless genetic exchange system (Keller et al., 2009), conceptual and predictive models of gene regulation and signal transduction (Rajeev et al., 2011; Turkarslan et al., 2017) and mapped networks of protein-protein interactions (Shatsky et al., 2016a, 2016b). Despite these advances, there remains considerable gaps in our understanding of $\mathrm{DvH}$, and, hence, SRB as a whole.

Transposon mutagenesis is a powerful genetic tool for generating a large collection of mutant strains, and the measured phenotypes of these strains can be used to infer gene functions. In SRB, an ordered transposon library has been generated and characterized in Desulfovibrio alaskensis G20 (Kuehl et al., 2014). This collection was subsequently used to gain new insights into the electron transfer complexes of this bacterium (Meyer et al., 2014; Price et al., 2014). In addition, the transposon insertion sequencing approach (Tn-seq), whereby the abundance of thousands of mutants are assayed simultaneously through next-generation sequencing (van Opijnen and Camilli, 2013; van Opijnen et al., 2009), has been applied in the humanassociated Desulfovibrio piger to characterize its metabolic niche (Rey et al., 2013) and in DvH to measure

83 phenotypes under two conditions (Fels et al., 2013). In these previous Tn-seq studies, phenotypes were not

84 detected for most genes in the respective genomes, partly because of the limited number of conditions 85 assayed. More recently, the random barcode transposon-site sequencing (RB-TnSeq) approach has been 86 developed which simplifies the measurement of mutant phenotypes across many conditions (Price et al., 87 2018; Wetmore et al., 2015), through the use of barcode sequencing or BarSeq (Smith et al., 2009). 
89 In this work, we report the generation of an RB-TnSeq library in DvH and its use in generating a large 90 gene-phenotype map using the BarSeq fitness assay across 757 experiments. These experiments represent 91244 unique growth conditions including changes in respiratory and fermentative growth conditions, growth 92 with different nitrogen and essential nutrient sources, and growth during exposure to various stressors.

93 Through the investigation of this large gene-phenotype dataset in $\mathrm{DvH}$, we define the essential gene set of 94 this bacterium and derive specific new insights into its metabolism, regulation, and stress response. 


\section{RESULTS}

\section{DvH essential genes in the wild-type and $\Delta u p p$ backgrounds}

101 Genes with few or no transposon insertions are likely essential for viability under the growth conditions

102 used to select the mutations. To identify the essential gene set of DvH, we first generated five transposon

103 mutant libraries in the wild-type background on lactate-sulfate rich growth medium using a barcoded

104 variant of the Tn5-RL27 transposon (henceforth called Tn5) (Larsen et al., 2002). Across all libraries, we

105 generated 116 million Tn-seq reads with a $\operatorname{Tn} 5$ transposon insertion that mapped to the DvH genome, with

106 the median gene represented by 17,823 reads. We used a previously described approach that estimates

107 essential genes based on a number of criteria including gene length, insertion density, insertions in the

108 central portion of the gene (as insertions near the 5' and 3' ends may not disrupt the gene's function), and

109 gene uniqueness (as we cannot discriminate insertions in highly repetitive regions) (Price et al., 2018; Rubin

110 et al., 2015). In total, we identified 399 likely essential genes in the wild-type strain (Supplementary Table

111 1). Of these 399 genes, $322(81 \%)$ have reduced transposon insertion coverage in a prior DvH transposon-

112 sequencing experiment with the same growth medium (Fels et al., 2013).

114 We next constructed 24 Tn5 transposon mutant libraries in the JW710 strain background. Combined, we generated 154 million reads with a transposon and a mapped location of each in the DvH genome, with the median gene represented by 27,580 sequencing reads. JW710 contains a deletion of upp (DVU1025),

117 encoding uracil phosphoribosyltransferase, a component of the pyrimidine salvage pathway. JW710 has

118 been adopted as a commonly used base strain in which to perform counter-selection with resistance to 5-

119 fluorouracil, a toxic pyrimidine (Keller et al., 2009). Using the same criteria applied to the wild-type

120 transposon insertion data, we identified 436 likely essential genes in the JW710 background

121 (Supplementary Table 1), of which 380 were in common with the wild-type background. The 380 genes

122 that are shared between the two strains are a robust estimate of the essential gene complement of DvH and

123 are enriched in general cellular processes such as protein synthesis and cell envelope functions (Figure 1A).

124 To explore these genes further, we compared each to the Database of Essential Genes (DEG) (Zhang et al.,

125 2004), which contains experimentally determined essential genes for dozens of diverse bacteria (all are

126 non-SRB). We found that the majority of these 380 genes perform more general functions unrelated to

127 sulfate reduction, as 271 have homologs that have been identified as essential in non-SRB. Only 109 of the

128 DvH essential genes did not have a good homolog in DEG (Materials and Methods), and these include a 
number of well-known genes directly involved in sulfate reduction including $d s r A B$ (dissimilatory sulfite reductase) and sat (sulfate adenylyltransferase).

Among the 19 genes uniquely essential in the wild-type background (and not in the JW710 background), we did not find a clear biological pattern. We suspect that most of these genes are nearly essential regardless of genetic background, because 18 of the 19 are near our essentiality threshold in the JW710 data (Materials and Methods). Among the 56 genes uniquely essential in JW710, we found that the entire eight gene de novo UMP biosynthesis pathway was essential, while all of these genes are clearly dispensable in the wildtype background. These synthetic lethality results are consistent because DvH has two pathways to make $\mathrm{UMP}$, a de novo pathway and a salvage pathway (Supplementary Figure 1). Due to the absence of the salvage pathway in JW710 (through the $\Delta$ upp mutation), the de novo pathway becomes essential. Other differences in gene essentiality between JW710 and wild-type DvH in our study, or between gene essentiality in wild-type DvH in our study or in Fels et al. (Fels et al., 2013), could be affected by sequence differences between these strains. Laboratory-acquired mutations in DvH can lead to large phenotypic differences between closely-related strains (De León et al., 2017). To identify genetic variants, we sequenced the JW710 genome and compared these data to our transposon mapping data for our wild-type strain (Materials and Methods). A list of the genetic variants in our wild-type and JW710 strains relative to the reference genome is contained in Supplementary Table 2.

\section{A gene-phenotype map of DvH}

RB-TnSeq simplifies the measurement of mutant phenotypes across multiple experiments through deep sequencing of DNA barcodes that uniquely mark each strain in the library (Price et al., 2018; Wetmore et al., 2015). To facilitate the generation of a large DvH gene-phenotype map, we first constructed a RBTnSeq mutant library in the JW710 ( $\Delta u p p)$ strain. We chose JW710 as the base strain because this background can be used for introducing a second mutation into the library, thus enabling future studies of genetic interactions in DvH. Using Tn-seq, we linked 74,923 unique DNA barcodes to transposon insertions in the main chromosome, and 4,737 unique DNA barcodes to insertions in the DvH native plasmid, pDV1. On both the chromosome and megaplasmid, $\operatorname{Tn} 5$ insertions were relatively evenly distributed 157 (Supplementary Figure 2). To perform genome-wide mutant fitness assays, we compare the abundance of 158 DNA barcodes after growth selection (referred to as the condition sample) versus before (referred to as the

159 Time0 sample), represented as a $\log _{2}$ ratio. To calculate gene fitness scores, we use the weighted average 160 of the individual strain fitness values for mutants in that gene (Wetmore et al., 2015). In the JW710 library, 161 we used 15 independent insertion strains to calculate gene fitness scores for the median gene. Negative 162 gene fitness scores mean that the mutations in this gene made mutants less fit than the average strain in the 
library, while positive gene fitness scores indicate that the mutations in the gene were beneficial to the mutant in these growth conditions.

Using the JW710 RB-TnSeq library, we generated a large DvH gene-phenotype map by performing 757 genome-wide fitness assays that passed our quality control metrics (Wetmore et al., 2015). In each of these experiments, we assayed the fitness of 2,741 protein-coding genes, for a total of 2.07 million genephenotype measurements. (241 non-essential proteins do not have fitness values, most often because mutants in these genes are at low abundance in the Time 0 samples.) To systematically investigate the physiology of DvH, we assayed a diverse range of conditions including respiratory growth, fermentative growth, growth in the presence of different nutrients, and growth in the presence of different stressors. The complete list of experiments, with associated metadata, is available in Supplementary Table 3. These 757 experiments include 244 unique experimental conditions (the remainder are biological replicates). The DvH fitness dataset can be explored interactively at the Fitness Browser (fit.genomics.lbl.gov), which bundles multiple computational tools to aid in the elucidation of novel gene functions. To illustrate the data, we highlight a comparison of two conditions, growth in defined media with either L-lactate or D-lactate as the sole carbon source and electron donor. As expected, many genes involved in the biosynthesis in amino acids had large fitness detects in both conditions (Figure 1B), while only a few genes had phenotypes unique to each substrate. In support of previous observations (Vita et al., 2015), our data demonstrates that DVU3032:DVU3033 encodes L-lactate dehydrogenase while DVU3027:DVU3028 encodes D-lactate dehydrogenase, as these genes have large growth defects on each substrate (Figure 1B). In addition, we found that DVU3031 is also important for growth on L-lactate. DVU3031 encodes a conserved but experimentally uncharacterized protein with AAA and DRTGG domains, and our data provides the first experimental evidence of the importance of this gene for growth on L-lactate, although its precise function remains to be determined.

Across the entire fitness dataset, we identified a significant phenotype (|fitness $\mid>0.5$ and $|t|>4$, where $t$ is a measure of the significance of the measurement (Wetmore et al., 2015)) for 1,137 genes in at least one experiment (at an estimated false discovery rate of 3\%; Materials and Methods). While non-essential genes from all functional categories (main roles from TIGRFAMs (Haft et al., 2013)) had significant phenotypes, those involved in general cellular processes, amino acid biosynthesis, and transport were more likely to

193 have a phenotype in one of our experiments, while those involved in mobile and extrachromosomal element 194 functions, regulation, and the cell envelope were less likely to have a phenotype (Figure 1A). Among non195 essential proteins with vague or hypothetical annotations, we identified a conditional phenotype for $34 \%$. 196 Our dataset provides a starting point for uncovering the roles for these genes. 
In our prior work, we have used two primary strategies to infer gene functions from mutant phenotypes: specific phenotypes and cofitness (Price et al., 2018). A gene has a specific phenotype if it has a phenotype in only one or a handful of conditions (Materials and Methods), in contrast to genes with more pleiotropic effects. Intuitively, specific phenotypes are informative for gene function because hypotheses can be readily derived from the one or few conditions where a phenotype is observed. For example, we found that DVU0599 (cstA) had a specific phenotype under conditions where pyruvate was used as the sole carbon source (Figure 1C), suggesting that this gene is involved in pyruvate utilization. DVU0599 is distantly related to Escherichia coli $\mathrm{YjiY}$ (32\% amino acid identity), which was recently demonstrated to be a pyruvate transporter (Kristoficova et al., 2018). The pyruvate-specific phenotype of DVU0599 strongly suggests that it also encodes a pyruvate transporter. We also identified pyruvate-specific phenotypes for the nearby two component signaling system encoded by DVU0596:DVU0597 (Figure 1C), which is consistent with the known regulation of DVU0599 by this system (Rajeev et al., 2011). Lastly, we found a similar pyruvate-specific phenotype for DVU2425 ( $\operatorname{rarD})$, which encodes an uncharacterized protein conserved in diverse bacteria (Figure 1C). RarD-family proteins are predicted to be transporters, and thus it is possible that DVU2425 also transports pyruvate. However, in other bacteria with available fitness data (Price et al., 2018), RarD proteins are important for transporting various amino acids. Across the entire dataset, we identified specific phenotypes for 540 different genes. These specific phenotypes are spread across a wide range of different experimental conditions, suggesting that they are useful for understanding different aspects of DvH biology.

Genes that have high cofitness ( $r$, correlated patterns of phenotypes across all experiments) are likely to share a cellular function (Deutschbauer et al., 2011; Price et al., 2018). For example, the phosphotransferase system (PTS) proteins PtsI (DVU0829) and DVU1632 (a putative EII-A enzyme) are highly cofit ( $r=0.89$ ), with both genes sharing fitness defects in a number of experiments with alternative nitrogen sources (Figure 1D). In the entire DvH dataset, we identified 2,104 gene pairs that have high cofitness $(r>=0.8)$, with 330 different genes having at least one cofitness relationship. In the subsequent sections, we combine the specific phenotypes and cofitness relationships with comparative genomics to derive new insights into the

225 functions of poorly understood $\mathrm{DvH}$ genes.

\section{$227 \quad$ DVU0867 encodes an atypical L-aspartate decarboxylase}

228 To identify genes that are required for vitamin biosynthesis, we tested growth in minimal medium with 229 vitamins omitted. (Our defined medium usually contained Thauer's vitamins.) To stimulate growth and 230 hence vitamin requirements, we added vitamin-free casamino acids (a mixture of amino acids) to the media 
for many of these experiments. We will describe novel findings in the biosynthesis of pantothenic acid (vitamin $\mathrm{B}_{5}$ ), biotin (vitamin $\mathrm{B}_{7}$ ), and pyridoxal phosphate (vitamin $\mathrm{B}_{6}$ ).

First, as shown in Figure 2A, we identified three genes that were specifically important when pantothenic acid was not available. Two of these genes (DVU2446 and DVU2448) were already annotated as being involved in pantothenic acid biosynthesis (panB and panC, respectively). The remaining gene, DVU0867, was annotated as aromatic amino acid decarboxylase. DVU0867 is distantly related (27\% amino acid identity) to the aspartate decarboxylase (PanP) of Vibrio fischeri (Pan et al., 2017). Aspartate decarboxylase is expected to be required for synthesis of pantothenic acid (Figure 2B). E. coli and many other bacteria encode $p a n D$ for this step, but neither panD nor its maturation cofactor panZ are present in $\mathrm{DvH}$ genome. So, we hypothesized that DVU0867 encodes an aspartate decarboxylase.

To test if DVU0867 could decarboxylate L-aspartate, we attempted to complement mutants of E. coli that require pantothenic acid for growth. The expression of DVU0867 restored growth of the panD mutant in the absence of pantothenic acid (Figure 2C), but did not restore growth of panC or panB mutants (data not shown). This confirmed that the protein encoded by DVU0867 performs the same function as PanD, Laspartate decarboxylase.

\section{Biotin synthesis with a specialized acyl carrier protein and without methyl esters}

Biotin, commonly known as vitamin $\mathrm{B}_{7}$, is an essential enzyme cofactor required by all three domains of life. However, it remains unclear how DvH synthesizes biotin, as it does not contain clear homologs for the entirety of known biosynthetic pathways. Because biotin is only required in trace amounts, we added the protein avidin $(0.1 \mathrm{U} / \mathrm{mL})$, which has a high affinity for biotin and sequesters it, to our no-biotin experiments. We identified ten genes that were specifically important for growth in the absence of biotin (Figure 3A). Nine of these ten genes were clustered together (DVU2558:DVU2565). This cluster includes bioF, bioA, bioD, and bioB, which together convert pimeloyl-[ACP] to biotin. (ACP is short for acyl carrier protein.) These genes bracket a cluster of genes encoding homologs to known fatty acid biosynthesis factors: DVU2560 and DORF42491 have FabZ-like domains, DVU2561 is a putative 3-oxoacyl-ACP reductase, DVU2562 is homologous to acyl-carrier protein, and DVU2563 contains a beta-keto-acyl carrier protein synthase (KAS) domain and is annotated as a FabF protein. These genes have previously been suggested to play a role as an alternate pathway for the synthesis of pimeloyl-ACP from malonyl-CoA,

262 which is the first stage of de novo biotin synthesis (Lin and Cronan, 2011; Rodionov et al., 2004). The 263 phenotype observed in our fitness assays are the first experimental evidence supporting their role in 264 synthesizing biotin. Another gene in the primary biotin synthesis cluster, birA (DVU2557) encoding a 
transcriptional repressor and biotin-protein ligase, did not have a strong phenotype in our no-biotin experiments. This is possibly due to the presence of another putative biotin-protein ligase (DVU1835) in the genome.

The single gene outside of this gene cluster whose mutant displayed a specific fitness defect in the absence of biotin was DVU1220, which is annotated as a nitroreductase. DVU1220 is predicted to contain both flavin mononucleotide (FMN) and [4Fe4S] iron sulfur cluster cofactors. We monitored the growth of the

272 parental strain, JW710, alongside DVU1220 and DVU2558 (bioB) mutants in media with and without biotin

273 depletion (Figure 3B). Both DVU1220 and DVU2558 mutants displayed significant growth defects under

274 biotin depletion conditions, which confirms a role for DVU1220 in biotin synthesis.

The existence of an extended biotin gene cluster was previously reported to be limited to the genus Desulfovibrio (Rodionov et al., 2004); (Lin and Cronan, 2011). More genome sequences are now available and we found similar gene clusters in two other orders of Deltaproteobacteria and in Desulfurispirillum indicum from the phylum Chrysiogenetes (Figure 3C, Supplementary Figure 3). Furthermore, in the genomes of Syntrophobacter fumaroxidans MPOB and Desulfobacter vibrioformis DSM 8776 for instance, these gene clusters include a nitroreductase-like gene (Figure 3C, Supplementary Figure 3). The nitroreductases in these biotin clusters are similar to each other (over $40 \%$ pairwise identity) but are distantly related to DVU1220; nevertheless, their clustering with the other genes of the pathway is consistent with the involvement of a nitroreductase-like protein in biotin synthesis in these organisms.

Although our data show that the entire biotin synthesis cluster as well as DVU1220 are required for biotin synthesis in DvH, further study will be needed to define the biochemical pathway for the first stage of biotin synthesis, up to pimeloyl-ACP. It appears that DVU2562 encodes an alternate ACP that is specialized for this pathway. (The other ACP in the genome, DVU1205, is essential, presumably because it is required for fatty acid biosynthesis.) In contrast to E. coli, where the enzymes for fatty acid biosynthesis convert malonyl-ACP methyl ester to pimeloyl-ACP methyl ester, it appears that DvH uses specialized enzymes to elongate malonyl-ACP to pimeloyl-ACP, without methyl ester intermediates. Indeed, neither the gene for forming the methyl ester (bioC) nor the gene for removing it (bioH) are found in $\mathrm{DvH}$. The elongation of malonyl-ACP to pimeloyl-ACP would require a $\beta$-keto-ACP synthase, a 3-oxo-ACP reductase, a $\beta$ -

295 hydroxyacyl-ACP dehydratase, and a enoyl-ACP reductase. The cluster contains candidates for all of these 296 activities except for enoyl-ACP reductase, which might be provided by the nitroreductase-like protein or 297 by a promiscuous enzyme from fatty acid biosynthesis (such as DVU2064, which is essential). 
By growing the mutant pool in defined medium that lacks pyridoxal phosphate (vitamin $\mathrm{B}_{6}$ ), we identified a putative two-subunit dehydrogenase (DVU0826 and DVU0827) that is required for pyridoxal phosphate biosynthesis (Figure 4A). The genes for both subunits have nearly identical fitness patterns (high cofitness) as $p d x A$ (DVU2241), which encodes 4-hydroxythreonine-4-phosphate dehydrogenase. Besides $p d x A$ and the dehydrogenase, the DvH genome also encodes pyridoxine 5'-phosphate synthase ( $p d x J, D V U 1908$ ), which implies that DvH synthesizes pyridoxal phosphate via deoxyxylulose 5'-phosphate, as in E. coli (Figure 4B) (Mittenhuber 2001; Fitzpatrick et al., 2007). The orthologs of DVU0826:DVU0827 in $D$. vulgaris Miyazaki F (DvMF_2874:DvMF_2875) display their highest co-fitness values $(r=0.78$ and 0.87 , respectively) with PdxJ (DvMF_0281) (data from Price et al 2018). This evidence supports a role for the dehydrogenase in pyridoxal phosphate biosynthesis.

As shown in Figure 4B, the DvH genome seems to be missing genes for two dehydrogenase enzymes in pyridoxal phosphate biosynthesis: 4-phosphoerythronate dehydrogenase ( $\mathrm{PdxB}$ in E. coli) and pyridoxine 5'-phosphate oxidase (PdxH in E. coli). DVU0921 encodes a pyridoxamine 5'-phosphate oxidase domain (PF12900), but this putative protein is very distantly related to $\mathrm{PdxH}$, and insertions in DVU0921 exhibited little phenotype in any of our assays (all |fitness $\mid<1$ ), so we do not think it encodes the missing PdxH. $P d x H$ is essential for the growth of most bacteria in media that contain yeast extract (data of Price et al 2018) because $\mathrm{PdxH}$ is required to convert pyridoxine to pyridoxal phosphate. Since DVU0826:DVU0827 are not essential, we suspect that they encode a novel 4-phosphoerythronate dehydrogenase.

\section{Utilization of nitrogen sources}

321 DvH is capable of fixing nitrogen gas (Heidelberg et al., 2004; Riederer-Henderson and Wilson, 1970) but 322 its capacity to use other nitrogen sources in the presence of pDV1-encoding nitrogenase has not been 323 reported. As far as we know, DvH does not use amino acids as the sole source of carbon for growth. We 324 assayed gene fitness for DvH in defined lactate-sulfate media with 28 different nitrogen sources, including 325 with $\mathrm{N}_{2}$ only. As expected, mutations in the nifD and nifK genes, encoding the alpha and beta subunits of 326 the nitrogenase complex respectively, were highly detrimental to growth when $\mathrm{N}_{2}$ was the sole nitrogen 327 source available, but had little effect on growth in ammonium (Figure 5). All of the genes in the nitrogen 328 fixation cluster (DVUA0007:DVUA0016) were important for growth with no added nitrogen, except for 329 DVUA0010, which had no fitness data. (After averaging across six replicate experiments with $\mathrm{N}_{2}$ as the 330 nitrogen source, each other gene in the cluster had fitness $<-2$.) 
331 We used the fitness of the nitrogenase genes nifDK to identify additional conditions under which nitrogen

332 fixation contributed to growth. We considered the nitrogen sources we tested as well-utilized if nifDK did

333 not contribute to fitness, and as weakly utilized if nifDK had a milder phenotype. Based on the fitness data

334 of nifDK, we found that among the amino acids glutamine and asparagine were well-utilized, and that

335 serine, tryptophan, and phenylalanine were weakly utilized (Figure 5). In addition, we found that growth in

336 minimal medium with no ammonium was stimulated by the addition of glutamine, asparagine, serine, or

337 phenylalanine (data not shown), which further confirms that these amino acids are utilized as nitrogen

338 sources by DvH. No growth after five days was observed when histidine, aspartate, or tyrosine were

339 provided as alternatives to ammonium. Growth occurred in the presence of the remaining 11 amino acids,

340 but appears to depend entirely on nitrogen fixation (Figure 5), which suggests that they are not utilized by

$341 \mathrm{DvH}$ as nitrogen sources. We did find that the addition of valine, isoleucine, leucine, or glutamate slightly

342 reduced the initial lag phase when DvH was grown in minimal medium with no ammonium (Supplementary

343 Figure 4). Nevertheless, because nitrogen fixation genes were important in these conditions, and because

344 we did not identify potential catabolic genes or transporters that were important for utilizing these four

345 amino acids, we believe that they are not utilized. The simulation of growth could be due to effects on gene

346 regulation or due the uptake of small amounts of amino acids.

347 The strong fitness defect of mutants in the asparaginase encoded by $D V U 2242$, when asparagine was used as sole nitrogen source (Figure 5), shows that this enzyme can efficiently provide ammonium. We did not identify any enzymes that were specifically important during growth on glutamine, so the origin of the glutaminase activity remains unclear. We did identify a putative transporter of the NbcE family (TC 2.A.115, (Saier et al., 2016)) that was specifically important for growth on glutamine (DVU2773), so we propose that $D V U 2773$ encodes the glutamine transporter. The putative serine dehydratase (DVU2147) and tryptophanase (DVU2204) were important for growth on serine and tryptophan, respectively (Figure 5), which confirms their participation in providing ammonium from these amino acids. We are not sure why both nitrogenase and a deaminating enzyme were important for growth with serine or tryptophan. It is possible that uptake is slow, that the deaminating enzymes are weakly expressed, or that nitrogen fixation and deamination are important during different phases of growth. We did not identify any genes that were specifically important for utilizing phenylalanine.

In addition to amino acids, we tested the utilization of polyamine, nucleobases, urea, nitrite, and formamide. In the presence of nitrite or formamide, nitrogen fixation was not required (Figure 5), which shows that DvH can utilize these nitrogen sources as well. As expected, nitrite utilization required the nitrite reductase

362 NrfA (DVU0625; Figure 5). Although the DvH genome contains a gene annotated as a formamidase 363 (DVU1164), we did not identify any phenotypes for this gene (all |fitness $\mid<0.5$ ). Overall, we found that 
DvH can utilize nine nitrogen sources (ammonia, $\mathrm{N}_{2}$, five of the amino acids, nitrite, and formamide), and we identified genes involved in the utilization of most of these nitrogen sources.

\section{Chlorate toxicity is mediated via the aldehyde oxidoreductase (Aor)}

The use of chlorate has been proposed as an additive to control the growth of SRB and concomitant sulfide production, which causes oil souring and is a major industrial problem (Engelbrektson et al., 2014; Gregoire et al., 2014). Previous work indicated that (per)chlorate can serve as specific and potent inhibitors of sulfate respiration (Carlson et al., 2015). It has been shown that both perchlorate and chlorate act, in part, as direct competitive inhibitors of sulfate adenylyltransferase, the first step in the pathway (Carlson et al., 2015;

372 Mehta-Kolte et al., 2019; Stoeva and Coates, 2019). Alternatively, reduction of (per)chlorate and their 373 conversion into reactive chlorine species (RCS) (chlorite and hypochlorite) has been attributed to the 374 adventitious reactivity of metal-binding pterin dependent enzymes such as nitrate reductase, which has a 375 molybdenum cofactor.

376 Although the DvH genome does not contain a predicted (per)chlorate reductase or nitrate reductase, the growth of DvH JW710 was significantly reduced when cultured in the presence of $10 \mathrm{mM}$ chlorate and was almost completely inhibited upon addition of $20 \mathrm{mM}$ chlorate (Figure 6A). Fitness profiling the chemogenomic response of JW710 to $6.25 \mathrm{mM}$ chlorate identified 15 genes that were detrimental to fitness (gave a positive fitness value when disrupted) in this condition but had little effect on fitness in the absence of chlorate (Figure 6A). Seven of these genes are involved in the biosynthesis of molybdenum cofactor or tungsten cofactor. (These are moaA (DVU0580), moaC (DVU0289), moaE (DVU2212), moeA (DVU2990), moeA-2 (DVU0951), moeB (DVU0643), and mogA (DVU0971)). Another four genes are involved in the uptake of tungstate (tupABC or DVU0747:DVU0745) or its regulation (tupR or DVU3193; (Rajeev et al., 2018)). The gene with the largest effect on fitness was aor (DVU1179), encoding a putative tungstendependent aldehyde:ferredoxin oxidoreductase.

It appears that biosynthesis of the tungsten cofactor is detrimental to the parental strain in the presence of chlorate because it allows for the activity of Aor. Furthermore, this effect does not occur with other oxyanions of chlorine: mutants in aor and the other tungsten-related genes were about as sensitive as other mutants in the presence of perchlorate or chlorite. (Fitness values for all twelve of those genes when challenged with $6.25-12.5 \mathrm{mM}$ perchlorate or $0.1-0.25 \mathrm{mM}$ chlorite were between -1 and +1 across all three replicates of each condition.) To quantify the advantage of disrupting aor or tungsten cofactor biosynthesis genes during growth on chlorate, we compared the growth of mutant strains for aor, moeA, and moeA-2 to the library parental strain JW710 in presence of up to $20 \mathrm{mM}$ chlorate. All strains grew similarly in the absence of chlorate (Figure 6B); JW710 was inhibited by $10 \mathrm{mM}$ chlorate (Figure 6C); and 
the interruption of aor or of the tungsten cofactor biosynthesis genes conferred resistance to $20 \mathrm{mM}$ chlorate (Figure 6D).

Based on these data, we propose that Aor catalyzes the reduction of chlorate to chlorite, which is far more toxic. Thus, disruption of aor itself, or of various genes involved in the acquisition of tungstate or the biosynthesis of tungsten cofactor, will confer resistance to chlorate. To test if this mechanism of chlorate toxicity applies to other sulfate-reducing bacteria, we examined previously-published fitness data for $D$.

402 vulgaris Miyazaki F and D. alaskensis G20 growing in lactate-sulfate medium in the presence of chlorate.

403 In D. vulgaris Miyazaki F, the three most detrimental genes during growth in $6.25 \mathrm{mM}$ chlorate were all

404 involved in molybdenum or tungsten cofactor biosynthesis (fitness > +6.0, data of (Price et al., 2018)). The 405 ortholog of aor (DvMF_1956) showed strongly positive fitness (fitness $=+5.6)$ and two other 406 molybdopterin-containing enzymes predicted to be anaerobic dehydrogenases ( $D v M F_{-} 1484$ and $407 D v M F \_0448$ ) had milder positive fitness values (fitness $=+2$ and +1.5 respectively). This suggests that 408 several enzymes contribute to the reduction of chlorate in D. vulgaris Miyazaki F. In D. alaskensis G20, 409 the six genes providing functions that were most detrimental to growth in the presence of chlorate are all 410 involved in molybdenum or tungsten cofactor biosynthesis (data of (Carlson et al., 2015)). Thus, in other 411 sulfate-reducing bacteria, the activity of molybdopterin-dependent enzymes is involved in chlorate toxicity.

412 Our data also allow the inference that both moeA and moeA-2 are required for the formation of the tungsten 413 cofactor of aor. Furthermore, the two genes have high cofitness across all of our experiments $(r=0.85)$.

414 Their orthologs in D. vulgaris Miyazaki F ( $D v M F_{-} 1797$ and $\left.D v M F_{-} 1358\right)$ also have high cofitness $(r=$ 415 0.90). The two moeA-like proteins of DvH are distantly related (33\% identity) and DVU0951 (annotated as 416 MoeA) has an additional C-terminal domain (PF12727) from the periplasmic binding protein superfamily. 417 It is not understood why many bacterial genomes contain two moeA-like genes, unlike E. coli, which has a 418 single moeA. Some researchers have speculated that the two copies might be specialized for the insertion 419 of molybdate or tungstate (i.e., (Smart et al., 2009)), but this is not the case in Desulfovibrio, nor are the 420 two moeA genes functionally redundant since the inactivation of each resulted in resistance to chlorate.

421 The remaining three genes that are detrimental during growth of JW710 in $6.25 \mathrm{mM}$ chlorate form a putative 422 three-component signaling system that includes a Lon-type protease, a histidine kinase, and a DNA-binding 423 response regulator (DVU3303:DVU3305). The three genes have very similar fitness patterns (all pairwise 424 cofitness $>0.9$ ), which confirms that they function together. One of the regulatory targets of this system is 425 the putative anion transporter encoded by DVU3299 (Rajeev et al., 2011). DVU3299 is important for growth 426 in chlorate stress (average fitness $=-2.3$ across three replicates), so the phenotype of the signaling system 427 may be due to its effect on the expression of DVU3299.

\section{The response to molybdate toxicity}


Molybdate $\left(\mathrm{MoO}_{4}{ }^{2-}\right)$ is highly toxic to SRB due to its capacity to act as a futile substrate for the adenosine phosphosulfate (APS) reductase, a key enzyme in the sulfate reduction pathway (Peck, 1962). We examined the response of the JW710 mutant pool when challenged with $100 \mathrm{uM} \mathrm{Na} \mathrm{MoO}_{4}$ under lactate-sulfate,

433 pyruvate-sulfate and pyruvate-sulfite growth conditions using BarSeq. A total of 40 genes were found to

434 respond specifically to the presence of molybdate. Here we will highlight two gene clusters that were not

435 previously linked to molybdate and which exhibited among the strongest responses: DVU0436:DVU0438 436 and DVU0539:DVU0545 (Figure 7A).

437 First, mutations in DVU0436, encoding a TetR-type transcriptional regulator, displayed a fitness advantage 438 for the mutants in the presence of molybdate (Figure 7A). In contrast, strains lacking DVU0437 or 439 DVU0438, which are annotated as the membrane fusion protein (MFP) and the integral membrane subunits of a resistance-nodulation-division (RND) type efflux pump, had significant fitness defects in the presence of molybdate (Figure 7A). These data suggest that DVU0436 represses transcription of the RND-type efflux pump encoded by DVU0437:DVU0438. Indeed, the RegPrecise database predicts that DVU0436 regulates the operon DVU0436:DVU0438 via a site just upstream of DVU0436 (Novichkov et al., 2013).

RND efflux pumps have a third outer membrane component, but no candidates for the missing component were found near DVU0437:DVU0438. Instead, based on cofitness, we identified DVU3097 as the probable outer membrane component. DVU3097 shares the molybdate-related phenotypes of DVU0437:DVU0438 but also has other phenotypes (Figure 7B). The other phenotypes suggest that DVU3097 encodes the outer membrane component of other efflux systems as well. The orthologous efflux system in $D$. vulgaris Miyazaki F ( $D v M F_{-}$1516:DvMF_1515/DvMF_2365) is specifically important for growth in the presence of the antibiotic carbenicillin (data of (Price et al., 2018)). In both strains of Desulfovibrio, the efflux system is probably involved in maintaining cell wall integrity rather than in the efflux of molybdate or a related compound.

The second molybdate-responsive cluster, DVU0539:DVU0545, is comprised of two divergently transcribed operons, DVU0540:DVU0539 and DVU0542:DVU0545. (There is no DVU0541 gene.) DVU0540:DVU0539 encode a sensor histidine kinase and a DNA-binding response regulator, respectively. DVU0539 is thought to regulate both operons, and when cloned into E. coli, DVU0539 can activate transcription from DVU0542's promoter (Rajeev et al., 2011). DVU0542:DVU0545 encode two proteins with homology to universal stress proteins (DVU0542, DVU0545), a transporter from the putative sulfate exporter (PSE) family (DVU0543; TC 2.A.98; (Saier et al., 2016), and a 133-amino acid protein with a transmembrane helix (DVU0544). The histidine kinase (DVU0540) and one of the universal stress proteins (DVU0542) were important for growth in the presence of molybdate, while the other genes in the cluster 
were detrimental during growth in the presence of molybdate, resulting in a positive fitness value when disrupted (Figure 7A). The only exception was the response regulator (DVU0539) had no phenotype during molybdate stress in lactate-sulfate medium (Figure 7A). Based on these data, it appears that the histidine kinase DVU0540 opposes the activity of the response regulator DVU0539. Nevertheless, the roles of DVU0542:DVU0545 in responding to molybdate remain unclear and will require further investigation.

\section{CONCLUSION}

Despite the importance of SRB to many environmental and industrial processes, we still have a limited molecular genetic understanding of these bacteria relative to well-studied species such as E. coli. To increase our knowledge of SRB biology, we applied a high-throughput genetics driven approach, RB-

473 TnSeq, to systematically identify mutant phenotypes for the commonly studied SRB D. vulgaris

474 Hildenborough. From these phenotypes and comparative genomic analyses, we were able to make a number of new insights into the physiology and metabolism of DvH.

The large-scale genetic dataset we present for DvH can serve as a powerful tool for developing hypotheses regarding the functions of genes in this bacterium. Importantly, our dataset complements other resources available for this strain including gene regulatory and metabolic models. The DvH RB-TnSeq library in JW710 is readily available for other groups to perform additional genome-wide assays; furthermore the availability of archived single mutants (both targeted gene deletions and transposon insertion strains) can greatly accelerate follow-up studies on genes of interest.

\section{MATERIALS AND METHODS}

\section{Bacterial strains and materials}

488 The bacterial strains and oligonucleotides used in this study are listed in Supplementary Tables 4 and 5 , 489 respectively. Oligonucleotides for cloning were ordered from Life Technologies (www.thermofisher.com) 490 and oligonucleotides for next-generation sequencing libraries preparation were ordered from Integrated 491 DNA Technologies (www.IDT.com). We used the GoTaq® Green Master Mix (Promega) for colony PCRs; 492 and Q5 hot start DNA polymerase (New England Biolabs) for all other PCR reactions. DNA fragments 493 were purified with the QIAquick PCR purification or Gel extraction kit (Qiagen). T4 DNA ligase and buffer 494 were purchased from NEB. Plasmid and genomic DNA isolations were carried out with the QIAprep Spin 495 Miniprep Kit and the DNeasy Blood \& Tissue Kit (Qiagen), respectively. 
497 The DvH genome annotation used in this study includes protein-coding genes that are not in the current

498 version in GenBank (GCF_000195755.1). These additional genes were identified by transcriptomics and 499 proteomics evidence (Price et al., 2011), and each starts with the systematic name "DORF". These 500 annotations are included in the DvH information at MicrobesOnline (Dehal et al., 2010).

502 The genes neighborhood comparison shown in this study was achieved using the BioCyc Database 503 collection and the Ensembl Bacteria browser (Howe et al., 2020; Karp et al., 2019).

\section{Growth conditions}

506 E. coli conjugation strain APA766 was cultured in Luria-Bertani (LB) medium at $37^{\circ} \mathrm{C}$ supplemented with $507100 \mu \mathrm{g} / \mathrm{ml}$ kanamycin and diaminopimelic acid (DAP) added to a final concentration of $300 \mu \mathrm{M}$. D. vulgaris 508 Hildenborough strains were grown within an anaerobic chamber (Coy Laboratory Products, Grass Lake, $509 \mathrm{MI}$ ) with an atmosphere of about $2 \% \mathrm{H}_{2}, 5 \% \mathrm{CO}_{2}$, and $93 \% \mathrm{~N}_{2}$. DvH was grown in MO medium (Zane et 510 al., 2010). When $60 \mathrm{mM}$ lactate (carbon source), $30 \mathrm{mM}$ sulfate (electron acceptor) and yeast extract (1 $511 \mathrm{~g} /$ liter) were added, the medium was designated MOYLS4 medium. A description of the media used in this 512 study and their composition is given in Supplementary Table 6. Other carbon sources and electron acceptors 513 were used and their concentrations are detailed in Supplementary Table 3. All media were autoclaved, 514 moved to the anaerobic chamber before cooling and amended with sodium sulfide $(1 \mathrm{mM})$ as reductant prior 515 to inoculation. MOYLS4 agar plates were poured inside the anaerobic chamber 1 - 2 days prior to use. 516 Culture growth was measured with a Bioscreen C instrument (Growth Curves USA) housed within the 517 anaerobic chamber at $30^{\circ} \mathrm{C}$.

\section{Transposon mutant library construction}

520 We constructed Tn5 transposon mutant libraries in the wild-type and JW710 backgrounds with minor 521 modifications to those previously described protocols (Wetmore et al., 2015). Our Tn5 transposon was a 522 barcoded derivative of the Tn5-RL27 transposon (Larsen et al., 2002), which is itself a derivative of the 523 Tn5 transposon (Reznikoff, 2008). Briefly, we conjugated mid-log-phase grown E. coli APA766 and DvH 524 cells at 2:1 ratio on a $0.45 \mathrm{uM}$ nylon membrane filter (Supor) overlaid on MOYLS4 agar plates. After 4 525 hours of anaerobic incubation $\left(30^{\circ} \mathrm{C}\right)$, the filters were transferred into liquid MOYLS4 medium. After 4 526 hours of recovery at $30^{\circ} \mathrm{C}$, the cells were then transferred into the same medium supplemented with G418 527 (400 ug/mL), and grown to saturation to select for G418-resistant transposon mutants. We made multiple, 528 single-use glycerol stocks of the library and extracted genomic DNA for Tn-seq analysis. To map the 
genomic location of the transposon insertions and to link these insertions to their associated DNA barcode, we used the same Tn-seq protocol that was previously described (Wetmore et al., 2015).

\section{Identification of essential genes}

533 Genes that are essential (or nearly so) for viability under the conditions used to select the mutants were 534 identified with previously described criteria (Price et al., 2018; Rubin et al., 2015). Only protein-coding 535 genes with at least 100 nucleotides of non-repetitive sequence (so that transposon insertions could be 536 unambiguously mapped) were considered. A gene was deemed essential if the normalized density of 537 insertions (dens, scaled so that the median is 1) and normalized reads per kilobase (normreads, scaled so 538 that the median is 1 and also normalized for GC content) were both below 0.2 (see Supplementary Table 539 1), and if the mutants in that gene did not have sufficient abundance in Time0 samples to calculate fitness 540 scores. As the wild-type strain was not used for fitness assays, we used the Time0 samples from the JW710 541 mutant library for this parameter in the wild-type essentiality calls. Of the 19 genes called as essential in 542 the wild-type background but not in JW710, 18 were considered "nearly essential" because either dens or 543 normreads were below 0.2 (Supplementary Table 1). From the Fels et al. TnSeq data (Fels et al., 2013), we 544 determined the number of different positions with a central insertion (10 - 90\% of gene length; we ignored 545 insertion positions with only 1 read) and the total number of sequencing reads for each gene at these 546 positions (Supplementary Table 1). Genes with 10 or more insertion positions and 100 or more total reads 547 were considered to be dispensable for viability. To compare the 380 genes that were identified as likely 548 essential in both the wild-type and JW710 backgrounds to essential genes in other bacteria, we used the 549 Database of Essential Genes (Zhang et al., 2004), which contains lists of essential genes from dozens of 550 bacteria. Specifically, we downloaded the latest version of DEG (version 15.2) and used BLASTp to search 551 for homologs of the DVH proteins. We considered the DvH protein to have a homolog if percent identity 552 to the DEG protein was at least $30 \%$, and if the alignment covered at least $75 \%$ of the protein's length in 553 both the DvH and DEG proteins.

\section{Sequence differences between wild-type DvH and JW710}

556 The genome of JW710 was sequenced on an Illumina MiSeq by the University of Missouri DNA Core.

557 Within the Geneious software (v 8.1.9) and with default parameters, raw sequences from JW710 were 558 trimmed to remove adapter sequences, mapped to the DvH genome reference sequence (NCBI Accession 559 No. NC_002937.3 and NC_005863.1) with Bowtie2 (Langmead and Salzberg, 2012), and sequence 560 variants were identified. To identify sequence variants in the wild-type DvH strain used in this study, we 561 aligned the Tn-seq reads from the wild-type background (after removing the sequences corresponding to 
the transposon) against the published reference genome, and identified sequence variants as described above for JW710. This analysis covered about $75 \%$ of the genome.

\section{Genome-wide mutant fitness assays}

566 We performed pooled mutant fitness assays as described previously (Price et al., 2018; Wetmore et al., 567 2015). Briefly, an aliquot of the full transposon mutant library was inoculated in MOYLS4 medium 568 supplemented with G418 (400 ug/mL) and culture was left to grow anaerobically at $30^{\circ} \mathrm{C}$ until the cells 569 reached mid-log phase. Samples of the culture of pooled mutants were collected as the "Time0" controls. 570 The remaining culture was pelleted, washed twice with phosphate buffer, and finally resuspended in 571 phosphate buffer (or in MOLS4 when formate-acetate was used as only carbon source). We inoculated the 572 mutant pool in the selective medium at a starting optical density at $600 \mathrm{~nm}$ of 0.02 . The fitness assays were 573 grown in either 24-well microplates or in $15 \mathrm{~mL}$ tubes; all plates and tubes were equilibrated inside the 574 anaerobic chamber prior use. After 4 to 6 population doublings of the mutant library, we collected 575 "condition" samples. We then extracted genomic DNA from the Time0 and condition samples. The DNA 576 barcodes were amplified and sequenced (BarSeq) with previously established protocols (Price et al., 2018; 577 Wetmore et al., 2015).

\section{Data analysis}

580 We calculated gene fitness scores as described (Wetmore et al., 2015). Briefly, strain fitness scores are 581 calculated as the normalized $\log 2$ ratio of the abundance of the barcode after selection (condition) versus 582 before (Time0). Gene fitness is computed as the weighted average of the fitness of the individual mutants; 583 then, to correct for variation in copy number along the chromosome in growing cells, gene fitness values 584 are normalized so that the running median along the main chromosome is zero; finally, the gene fitness 585 values are normalized so that the mode (for genes on the chromosome) is zero. For each gene fitness score, 586 we calculate a $t$-like test statistic to determine the significance of the measurement (Wetmore et al., 2015). 587 For calculating the number of $\mathrm{DvH}$ genes with a significant phenotype in the entire dataset, we required $588 \mid$ fitness $\mid>0.5$ and $|t|>4$. This analysis identified 1,137 genes with a phenotype in at least one of the 757 589 experiments. To determine the false discovery rate (FDR), we used comparisons among the Time0 samples, 590 which are not expected to result in significant phenotypes. Among 83 Time 0 comparisons, we identified 4 591 genes with a significant phenotype with the same thresholds for fitness and $t$. After correcting for having 592757 condition experiments and only 83 Time0 samples, we estimated the FDR for genes with a significant 593 phenotype as $3 \%(36 / 1,137)$. 
DvH genes with a specific phenotype in an experiment were defined as: $\mid$ fitness $\mid>1$ and $|t|>5$; $\mid$ fitness $\mid<$ 1 in at least $95 \%$ of experiments; and the fitness value in this experiment was more pronounced that most of its other fitness values (|fitness $\mid>95$ th percentile $(\mid$ fitness $\mid)+0.5$ ) (Price et al., 2018). Cofitness was calculated as the Pearson correlation coefficient between all 757 fitness measurements for each pair of genes (Price et al., 2018).

600

601 To infer the functions of DvH genes based on mutant phenotypes, we primarily used the fitness browser 602 (fit.genomics.lbl.gov), which contains genome-wide mutant fitness data for 38 different bacteria and a 603 number of interactive tools for data exploration (Price et al., 2018). To determine the current state of 604 knowledge on individual DvH proteins and their homologs, we used PaperBLAST 605 (http://papers.genomics.lbl.gov/) (Price and Arkin, 2017). We used TIGRFAMs to assign DvH proteins to 606 different functional categories (primarily using main role categories) (Haft et al., 2013). RegPrecise 607 (Novichkov et al., 2013) was accessed using MicrobesOnline or from github 608 (https://github.com/SMRUCC/RegPrecise/tree/master/genomes).

To identify protein-coding genes with hypothetical or vague annotations, we matched their descriptions 611 against text patterns such as "hypothetical", "family", or "membrane protein," as described previously 612 (Price et al., 2018).

\section{Data and software availability}

616 The data and analyses described in this work can be accessed from different sources. First, the gene fitness

617 values and their comparison to fitness data from other bacteria are available through the fitness browser 618 (fit.genomics.lbl.gov, archived at https://doi.org/10.6084/m9.figshare.13172087.v1). This is the best 619 location to access the data to examine the phenotypes of specific genes of interest or to BLAST a protein 620 of interest against the entire dataset. Second, the gene fitness values, $t$ scores, detailed metadata for all 621 experiments, barcode counts, tables of genes with specific phenotypes and cofitness, and a single R image 622 with all analyzed fitness data, are available at figshare (https://doi.org/10.6084/m9.figshare.13010285).

623 Third, all of the TnSeq data is available from NCBI's sequence read archive under project PRJNA666215.

624 Fourth, genome sequencing data for strain JW710 is available at accession SRX9297596.

625

626 The software we used for analyzing the Tn-seq and BarSeq fitness data is available at 627 https://bitbucket.org/berkeleylab/feba/. 


\section{FIGURES}

A. Phenotypes by role

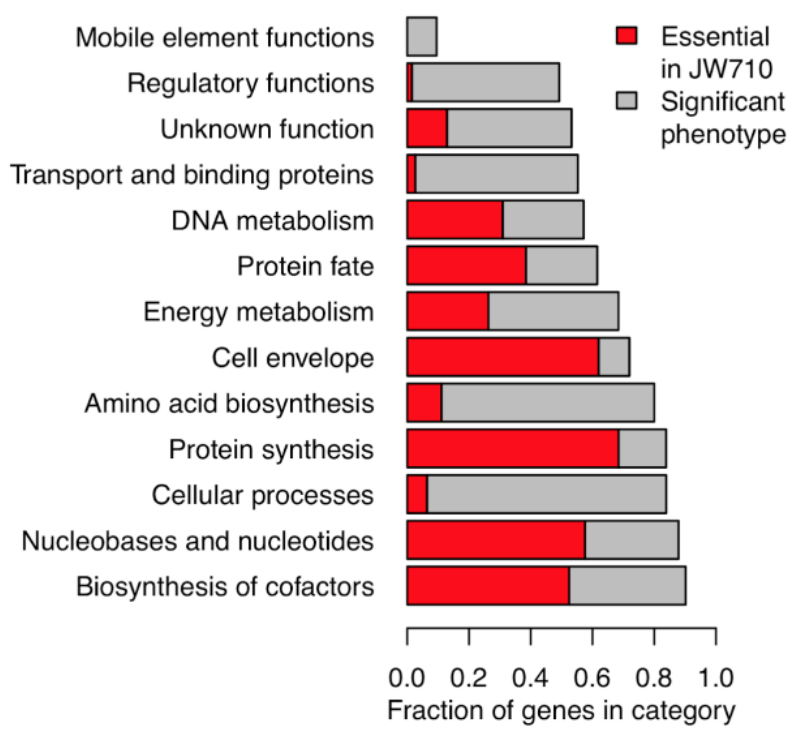

\section{Specific phenotypes on pyruvate}

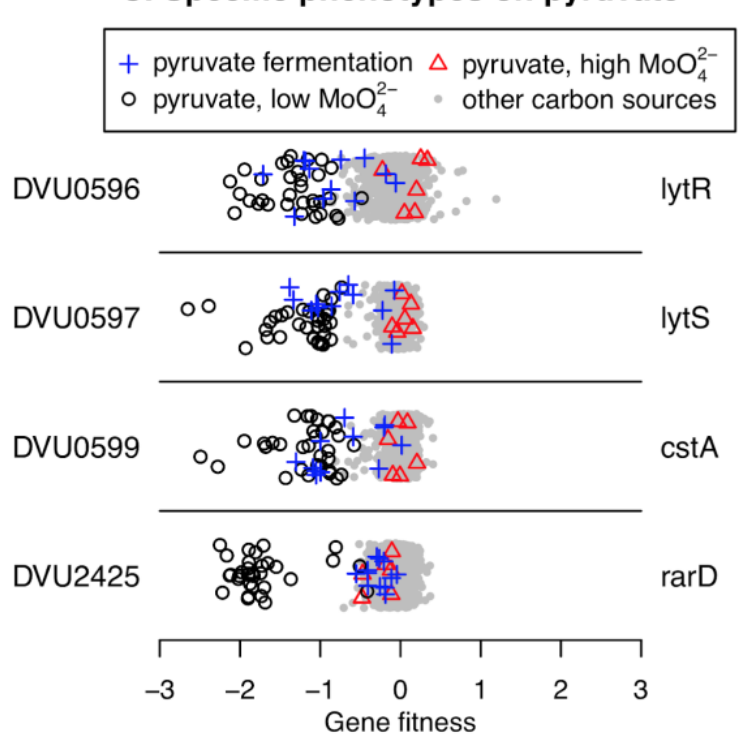

B. Lactate utilization

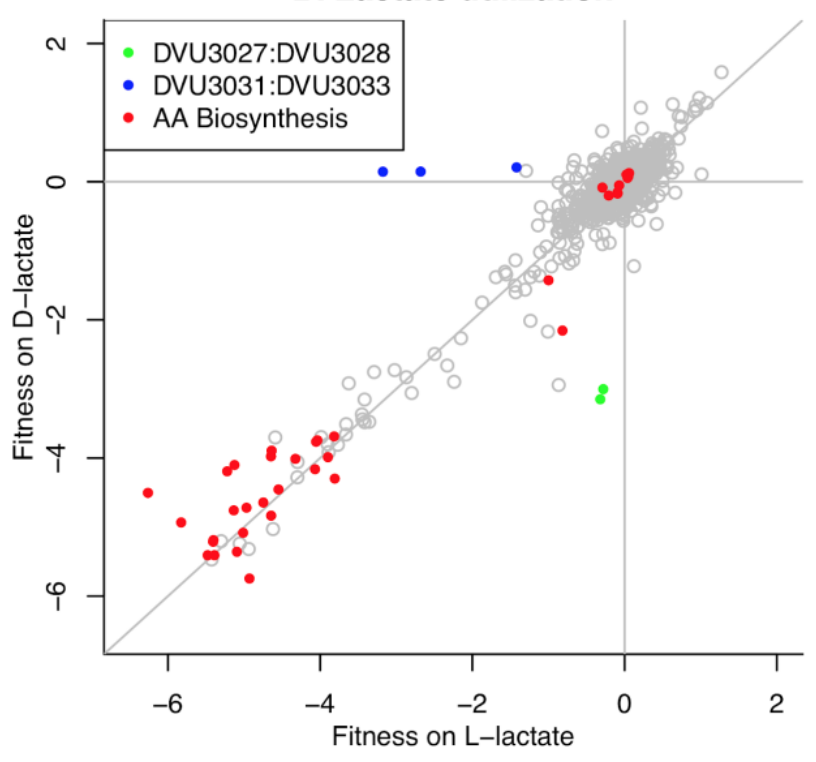

D. Cofitness of ptsI and DVU1632

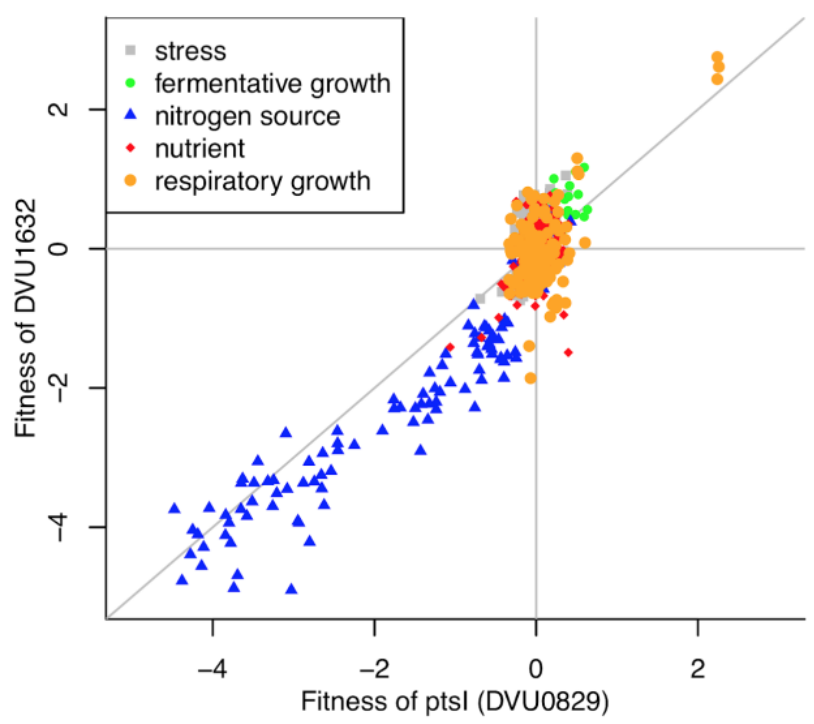

634 Figure 1. Summary of D. vulgaris Hildenborough mutant fitness dataset. (A) Fraction of genes from 635 different functional categories that were essential (in both wild-type and JW710 backgrounds) or had a 636 significant phenotype in at least one experiment. The functional categories are from TIGRFAMs roles (Haft 637 et al., 2013). Only categories with 20 or more genes are shown. (B) Comparison of gene fitness values for 638 growth in minimal media with either L-lactate ( $x$-axis) or D-lactate ( $y$-axis) as the carbon source. The data 
639 is the average of three biological replicates for each condition. "AA biosynthesis genes" are genes with

640 TIGRFAMs role of "Amino acid biosynthesis." (C) Specific phenotypes for genes important for growth on

641 pyruvate. In these plots, each point represents the fitness of that gene in one of 757 genome-wide assays.

642 Certain experiment classes are highlighted. The $y$-axis is random. (D) Comparison of fitness values for

643 DVU0829 ( $x$-axis) and DVU1632 (y-axis) across all 757 experiments. In panels B and D, lines show $x=0$, $644 y=0$, and $x=y$.

645

\section{A. Gene Fitness}

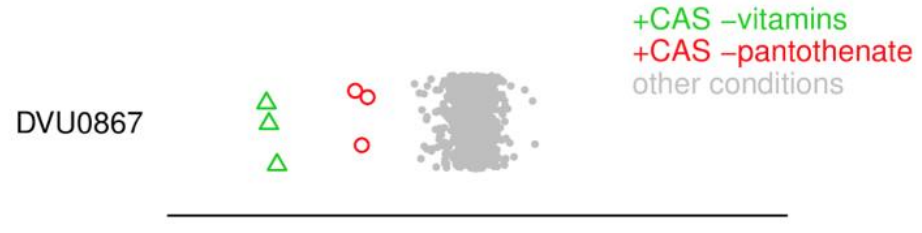

DVU2446

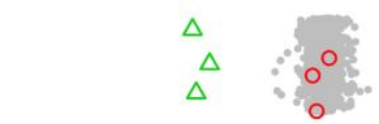

DVU2448 $\stackrel{\Delta}{\Delta}$
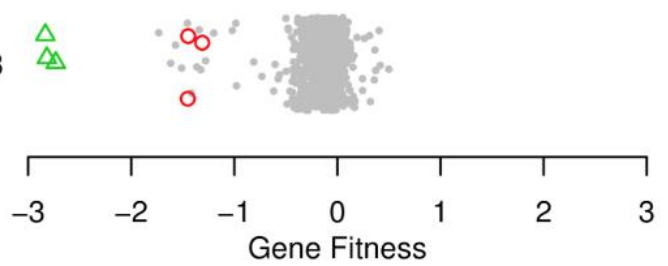

Gene Fitness

panB

panC

\section{B. Biosynthetic Pathway}

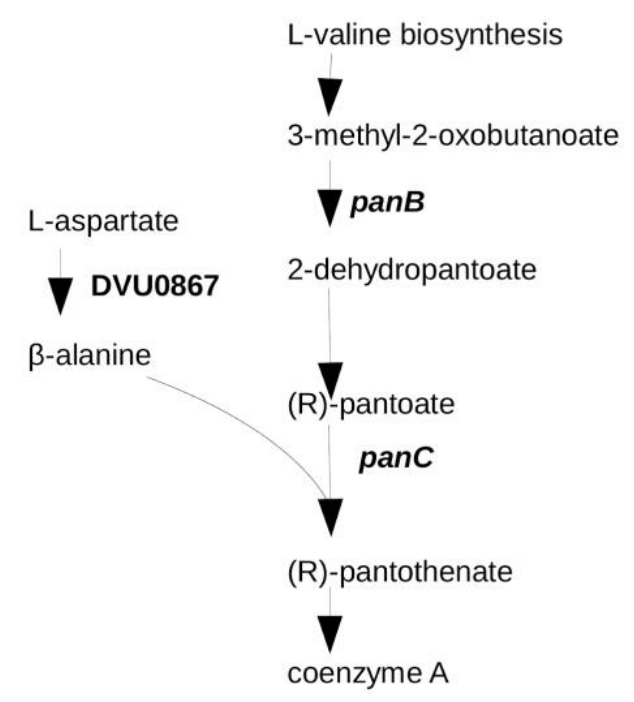

L-valine biosynthesis

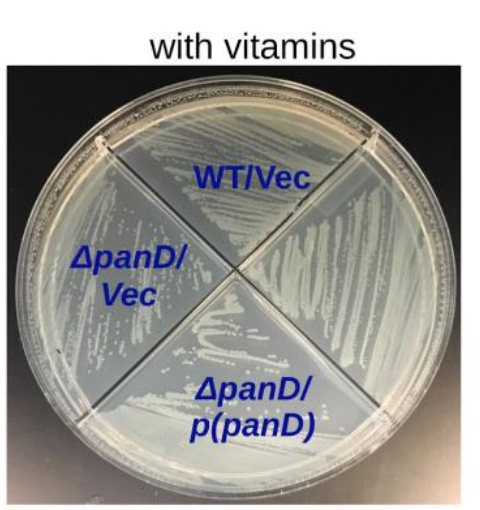

\section{Complementation Assay}

no pantothenic acid tarabinose

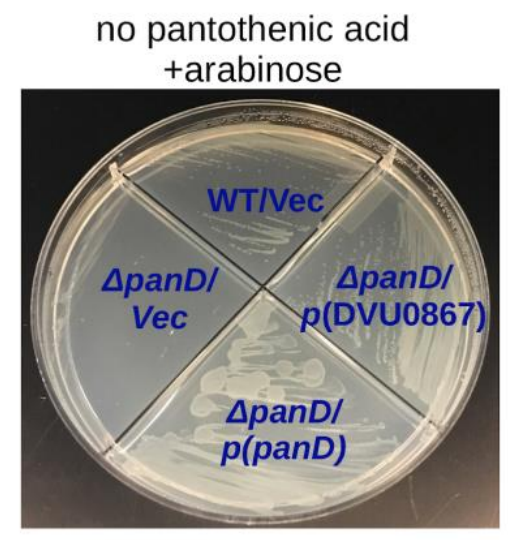

648 Figure 2. DVU0867 has L-aspartate 1-decarboxylase activity. (A) Fitness data for each gene across all 757 experiments, with pantothenate-related conditions highlighted. The $y$-axis is random and CAS is short for casamino acids. (B) The biosynthetic pathway for pantothenate, with key enzymes highlighted. (C) 
651 Complementation assays using an E. coli panD gene knockout strain that is defective in pantothenate

652 biosynthesis and lacks aspartate 1-decarboxylase activity. Indicated genes were cloned upstream of an

653 arabinose inducible promoter. Vec is an empty vector control.

654

\section{A. Gene Fitness}

+avidin -biotin other conditions

DVU2558

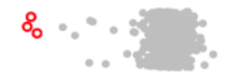

$\begin{array}{llll} & & & \\ \text { DVU2559 } & \circ & \therefore & \text { bioA }\end{array}$

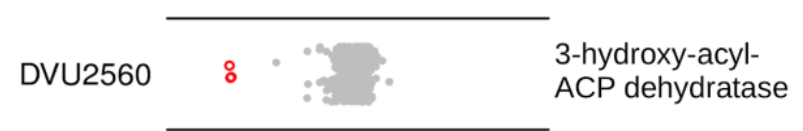

\begin{tabular}{lll}
\cline { 2 - 3 } DVU2561 & $\circ$ & $\begin{array}{l}\text { 3-oxoacyl-ACP } \\
\text { reductase }\end{array}$ \\
\cline { 2 - 3 } DVU2562 & $\circ$ & $\begin{array}{l}\text { acyl carrier protein } \\
\text { (ACP) }\end{array}$
\end{tabular}

$\begin{array}{llll}\text { DVU2563 } & \circ & \ddots & \begin{array}{l}\beta \text {-ketoacyl-ACP } \\ \text { synthase }\end{array}\end{array}$

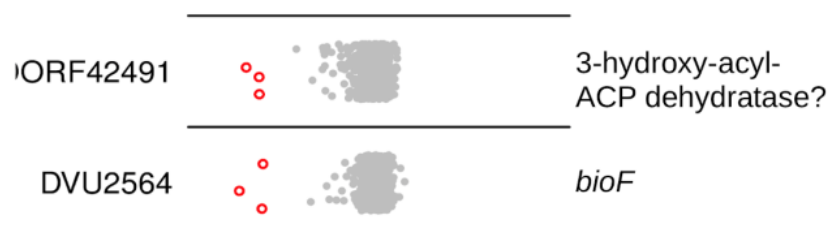

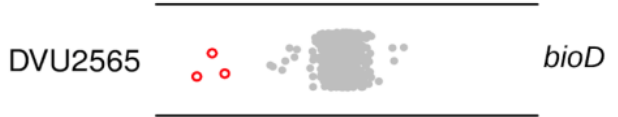

DVU1220

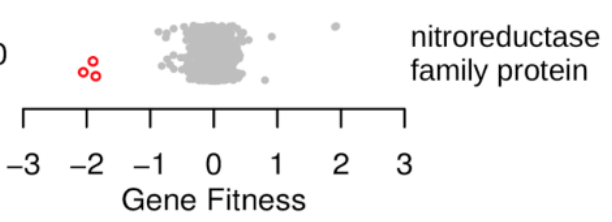

\section{B. Growth Curves}
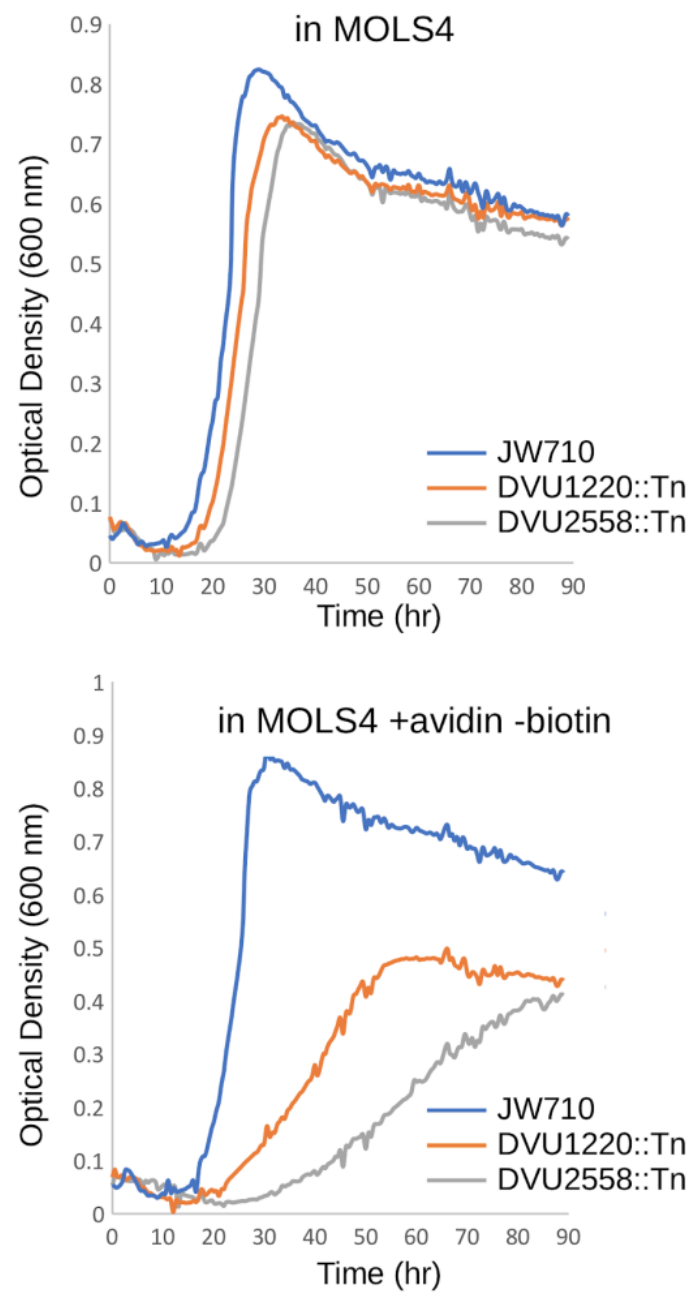

C. Gene Neighborhoods

Desulfovibrio vulgaris

DVU

25602561 acp 256342491 biof bioD

DVU1220

\section{Syntrophobacter fumaroxidans} (Syntrophobacterales)

Desulfobacter vibrioformis (Desulfobacterales)

\section{Desulfurispirillum indicum}


657 Figure 3. An atypical biotin synthesis pathway in D. vulgaris Hildenborough. (A) Fitness data for each 658 gene across all 757 experiments, with experiments in the absence of biotin and supplemented with avidin 659 highlighted. The $y$-axis is random. (B) Growth assays of the DvH JW710 control strain, and strains with 660 mutations in either DVU1220 or DVU2558 (bioB). Measurements were made in a Bioscreen C growth 661 analysis system and each curve is the average of four replicates. (C) Gene neighborhood and conservation 662 in other microorganisms with components of the DvH biotin synthesis pathway. Genes are colored by their 663 homology to the corresponding DvH genes, and birA is only shown if it is adjacent to other biotin synthesis 664 genes.

665 


\section{A. Gene Fitness}
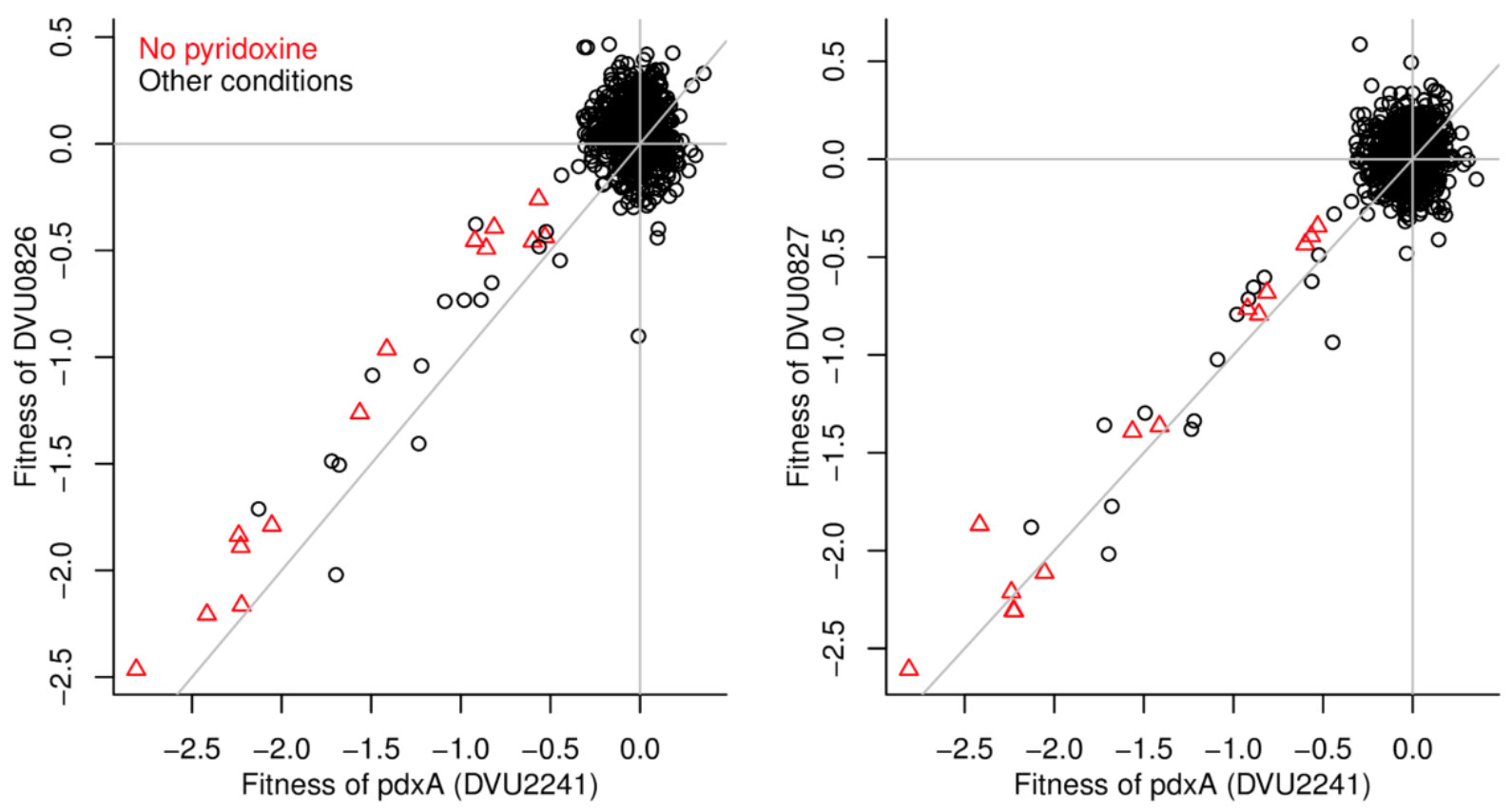

\section{B. Pathway}
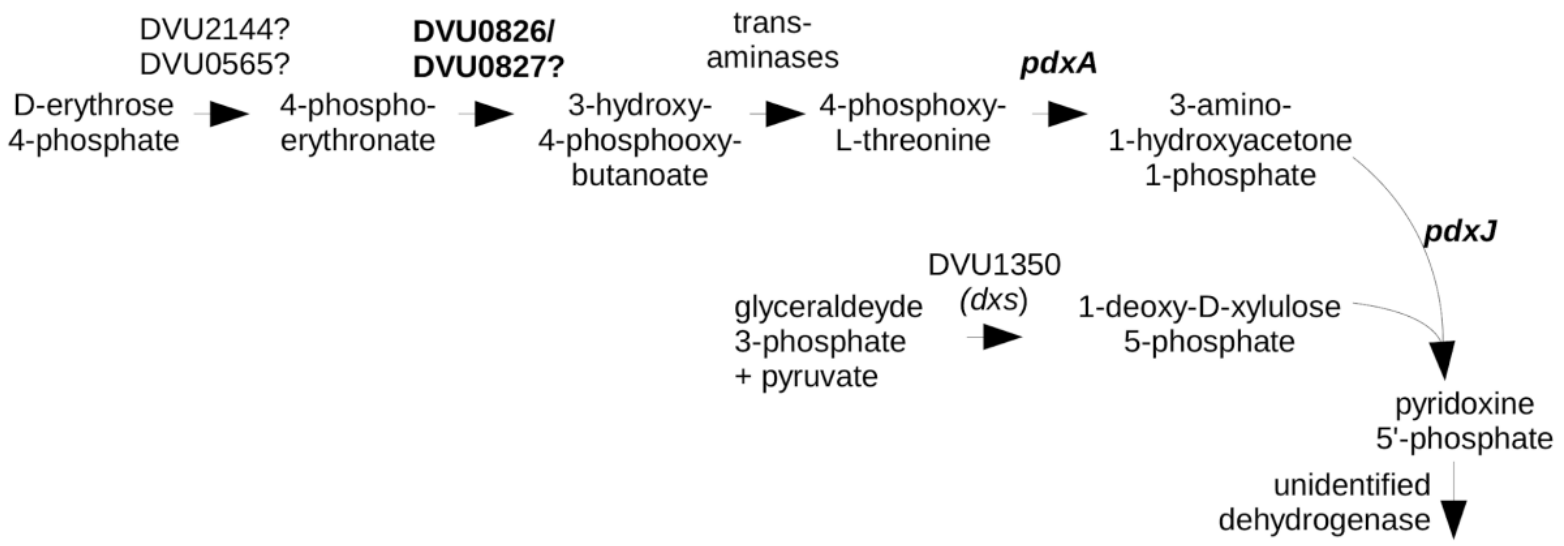

pyridoxal

5'-phosphate

668 Figure 4. DVU0826 and DVU0827 are required for vitamin $\mathrm{B}_{6}$ synthesis. (A) Comparison of gene fitness values across 757 experiments between $p d x A$ (DVU2241) and either DVU0826 (left) or DVU0827 (right). Experiments performed in the absence of pyridoxine are highlighted. $p d x A$ has high cofitness with both

671 DVU0826 $(r=0.81)$ and DVU0827 $(r=0.84)$. (B) The proposed pathway of pyridoxal phosphate 672 biosynthesis in DvH. 
bioRxiv preprint doi: https://doi.org/10.1101/2021.01.13.426591; this version posted January 13,2021 . The copyright holder for this preprint (which was not certified by peer review) is the author/funder, who has granted bioRxiv a license to display the preprint in perpetuity. It is made available under aCC-BY-ND 4.0 International license.

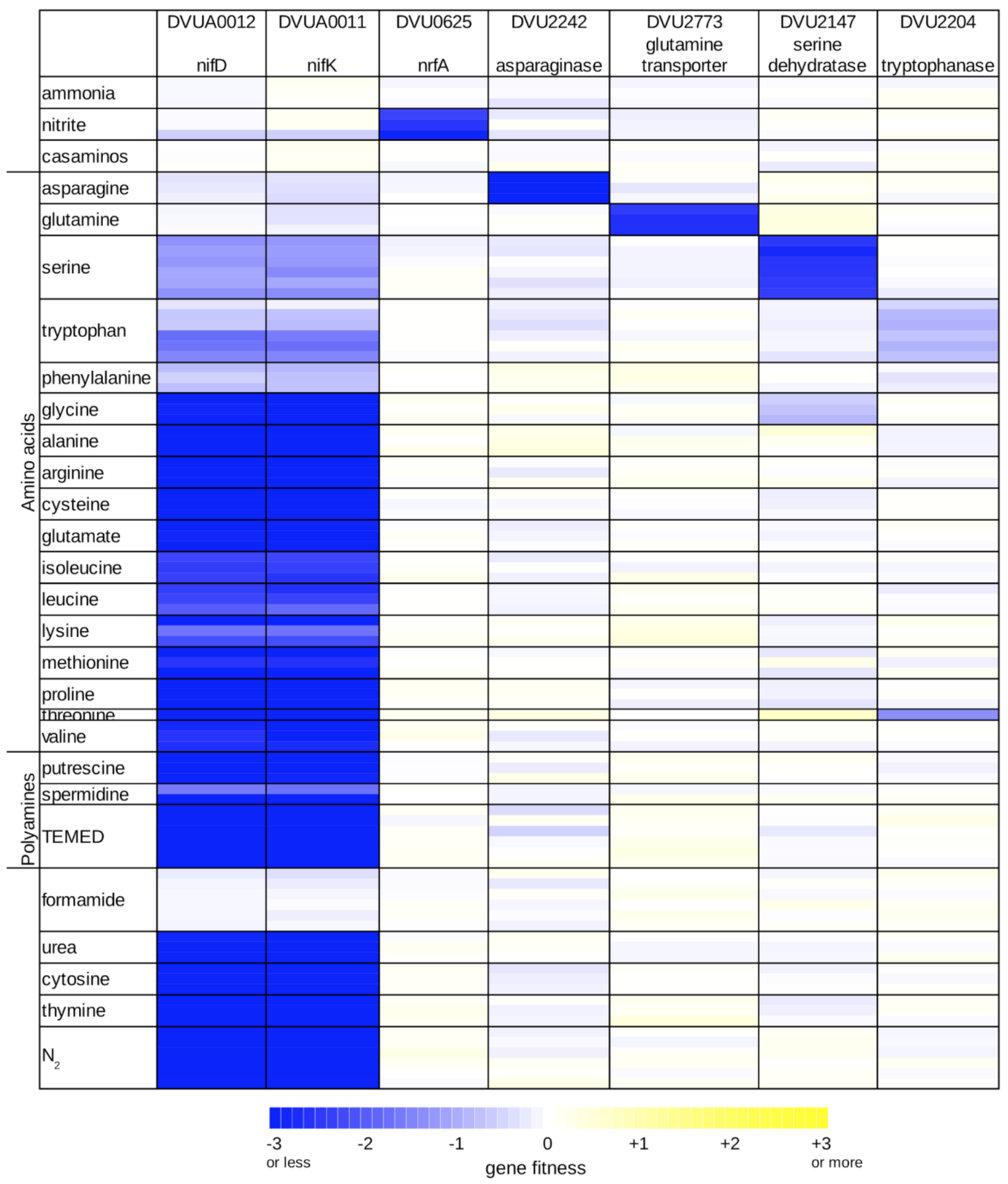

675 Figure 5. Overview of nitrogen utilization in D. vulgaris Hildenborough. Heatmap of gene fitness data for

676 select genes in experiments where the nitrogen source was varied. For each condition, we show the data

677 from each replicate experiment separately. TEMED is tetramethylethylenediamine; casaminos is casamino 678 acids. 


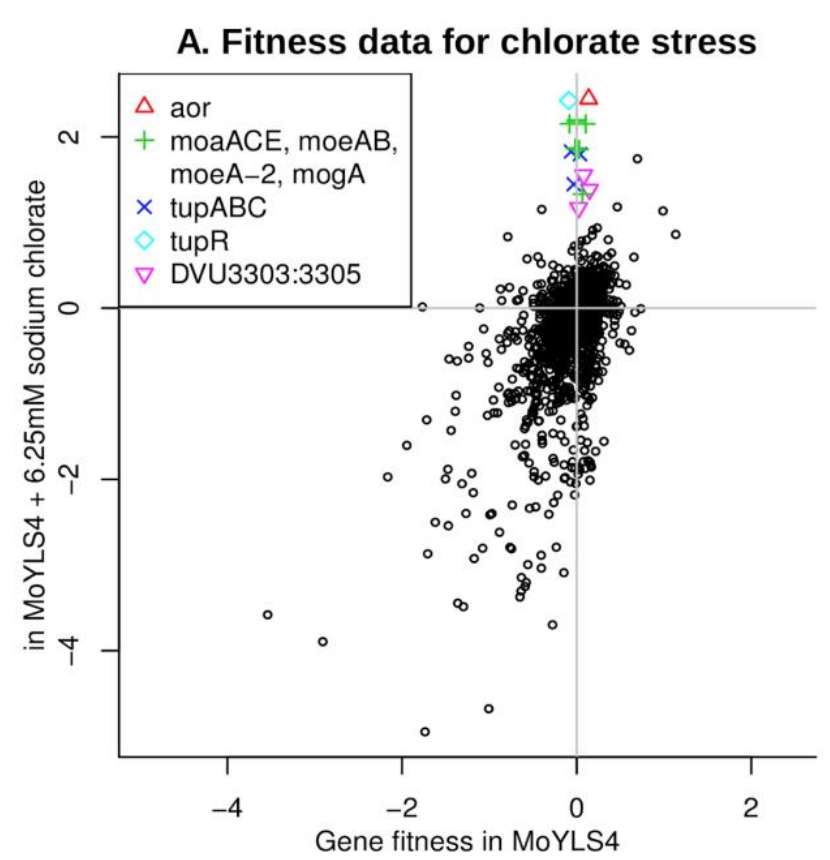

C. Growth in MoYLS4 + $10 \mathrm{mM}$ chlorate

680

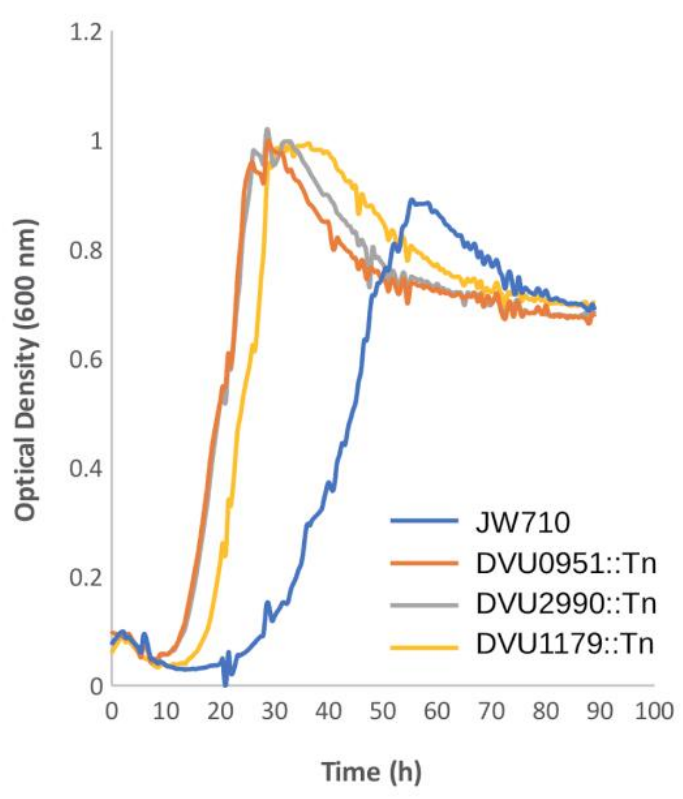

B. Growth in MoYLS4

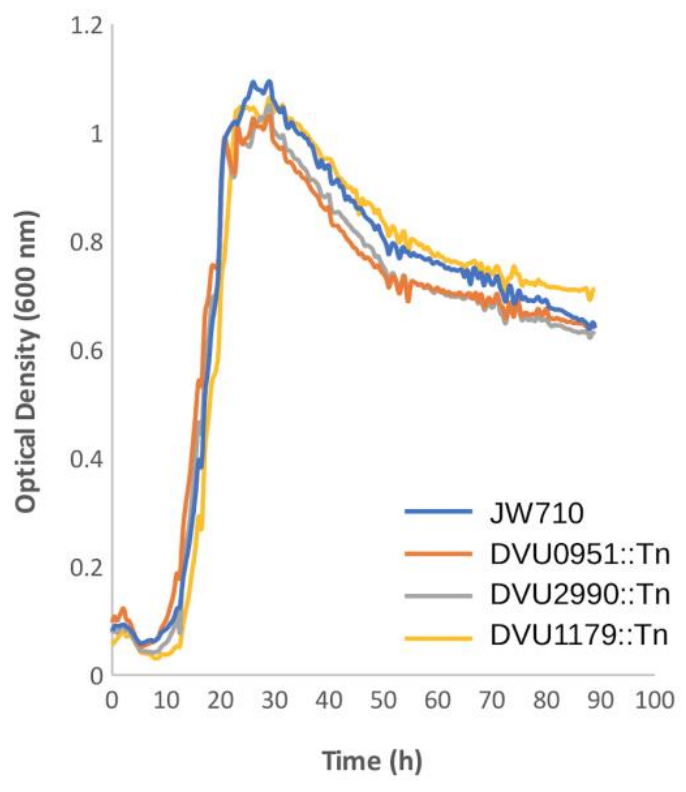

D. Growth in MoYLS4 $+20 \mathrm{mM}$ chlorate

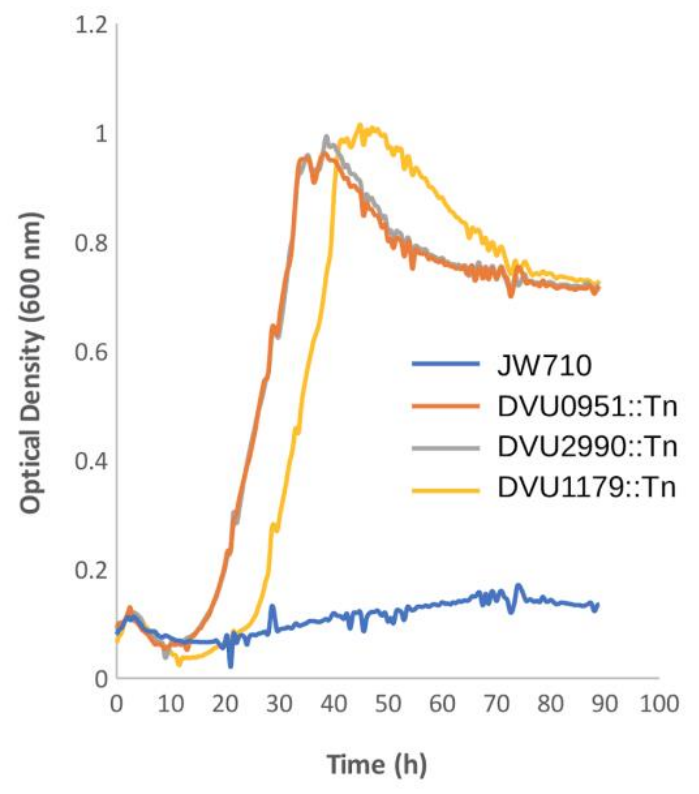

682 Figure 6. Loss of aldehyde oxidoreductase activity results in chlorate resistance. (A) Comparison of gene 683 fitness values for growth in rich lactate-sulfate media or in media supplemented with $6.25 \mathrm{mM}$ sodium 684 chlorate. Each value is the average from three replicate experiments. (B-D) Growth of DvH JW710 and mutant strains of DVU0951 (moeA), DVU2990 (moeA-2) and DVU1179 (aor) in rich media with increasing concentrations of sodium chlorate. Each curve is the average of four replicates. 


\section{A. Two molybdate stress clusters}

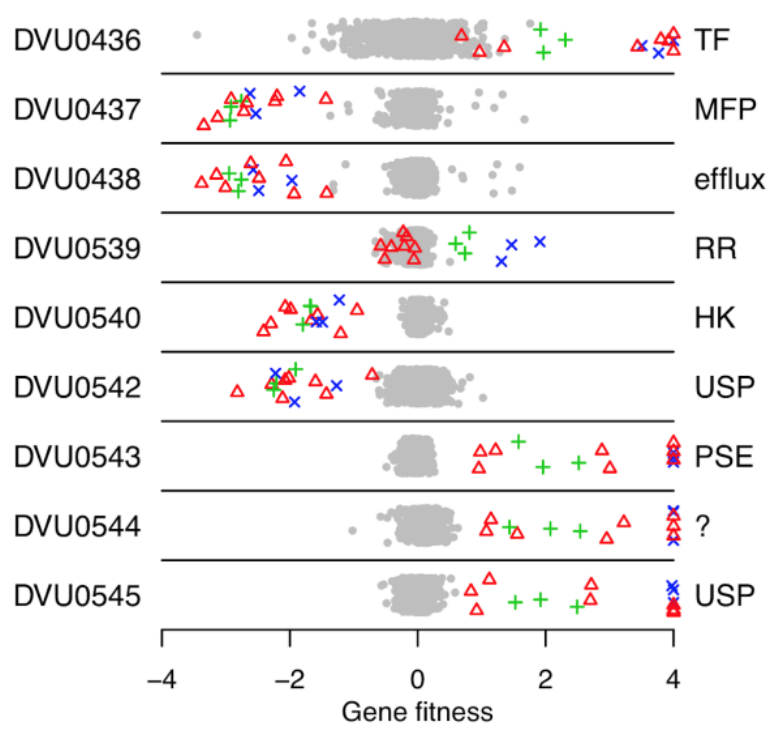

688

\section{B. DVU3097 is the OMP for DVU0437:DVU0438}

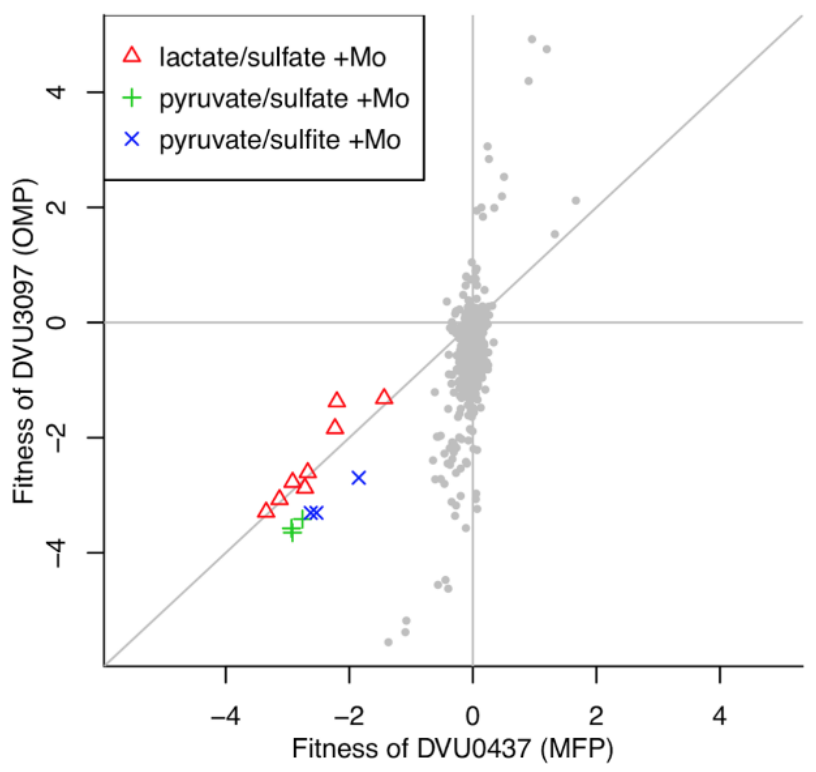

Figure 7. Selected genes with specific phenotypes during molybdate stress. (A) Two clusters of genes involved in molybdate stress. Each point represents the fitness of that gene in a genome-wide assay ( $x$-axis). Values above +4 are shown at +4 . The $y$-axis is random. (B) Comparison of fitness patterns for the membrane fusion protein DVU0437 and the outer membrane protein DVU3097. In both panels, experiments with added molybdate $(0.1 \mathrm{mM})$ are highlighted with the same color coding (see panel B legend). 4 of the 8 experiments with lactate/sulfate media and added molybdate also had tungstate added (at 0.5 or $2.0 \mathrm{mM}$ ).

\section{FUNDING SOURCES}

This material by ENIGMA- Ecosystems and Networks Integrated with Genes and Molecular Assemblies (http://enigma.lbl.gov), a Science Focus Area Program at Lawrence Berkeley National Laboratory is based upon work supported by the U.S. Department of Energy, Office of Science, Office of Biological \& Environmental Research under contract number DE-AC02-05CH11231. The funders had no role in study design, data collection and interpretation, or the decision to submit the work for publication. The United States Government retains and the publisher, by accepting the article for publication, acknowledges that the United States Government retains a non-exclusive, paid-up, irrevocable, world-wide license to publish or 
reproduce the published form of this manuscript, or allow others to do so, for United States Government purposes.

This work used the Vincent J. Coates Genomics Sequencing Laboratory at UC Berkeley, supported by NIH S10 OD018174 Instrumentation Grant.

\section{ACKNOWLEDGEMENTS}

We thank Lara Rajeev for her help with anaerobic growth monitoring and Hans K. Carlson for helpful 717 discussions and critical reading of the manuscript.

\section{REFERENCES}

Carlson, H.K., Kuehl, J.V., Hazra, A.B., Justice, N.B., Stoeva, M.K., Sczesnak, A., Mullan, M.R., Iavarone, A.T., Engelbrektson, A., Price, M.N., et al. (2015). Mechanisms of direct inhibition of the respiratory sulfate-reduction pathway by (per)chlorate and nitrate. ISME J. 9, 1295-1305.

De León, K.B., Zane, G.M., Trotter, V.V., Krantz, G.P., Arkin, A.P., Butland, G.P., Walian, P.J., Fields, M.W., and Wall, J.D. (2017). Unintended Laboratory-Driven Evolution Reveals Genetic Requirements for Biofilm Formation by Desulfovibrio vulgaris Hildenborough. MBio 8 . Dehal, P.S., Joachimiak, M.P., Price, M.N., Bates, J.T., Baumohl, J.K., Chivian, D., Friedland, G.D., Huang, K.H., Keller, K., Novichkov, P.S., et al. (2010). MicrobesOnline: an integrated portal for

731 Deutschbauer, A., Price, M.N., Wetmore, K.M., Shao, W., Baumohl, J.K., Xu, Z., Nguyen, M., Tamse, R., 732 Davis, R.W., and Arkin, A.P. (2011). Evidence-based annotation of gene function in Shewanella oneidensis 733 MR-1 using genome-wide fitness profiling across 121 conditions. PLoS Genet. 7, e1002385.

734 Engelbrektson, A., Hubbard, C.G., Tom, L.M., Boussina, A., Jin, Y.T., Wong, H., Piceno, Y.M., Carlson, 735 H.K., Conrad, M.E., Anderson, G., et al. (2014). Inhibition of microbial sulfate reduction in a flow-through 736 column system by (per)chlorate treatment. Front. Microbiol. 5, 315.

737 Fels, S.R., Zane, G.M., Blake, S.M., and Wall, J.D. (2013). Rapid transposon liquid enrichment sequencing 
(TnLE-seq) for gene fitness evaluation in underdeveloped bacterial systems. Appl. Environ. Microbiol. 79,

Gregoire, P., Engelbrektson, A., Hubbard, C.G., Metlagel, Z., Csencsits, R., Auer, M., Conrad, M.E., Thieme, J., Northrup, P., and Coates, J.D. (2014). Control of sulfidogenesis through bio-oxidation of H2S coupled to (per)chlorate reduction. Environ. Microbiol. Rep. 6, 558-564. genome properties in 2013. Nucleic Acids Res. 41, D387-95.

Heidelberg, J.F., Seshadri, R., Haveman, S.A., Hemme, C.L., Paulsen, I.T., Kolonay, J.F., Eisen, J.A., Ward, N., Methe, B., Brinkac, L.M., et al. (2004). The genome sequence of the anaerobic, sulfate-reducing bacterium Desulfovibrio vulgaris Hildenborough. Nat. Biotechnol. 22, 554-559.

Howe, K.L., Contreras-Moreira, B., De Silva, N., Maslen, G., Akanni, W., Allen, J., Alvarez-Jarreta, J., Barba, M., Bolser, D.M., Cambell, L., et al. (2020). Ensembl Genomes 2020-enabling non-vertebrate genomic research. Nucleic Acids Res. 48, D689-D695. (II), nickel (II), and chromium (VI) in a marine environment by using Desulfovibrio desulfuricans. Biotechnol. Bioprocess Eng. 20, 937-941.

754 Karp, P.D., Billington, R., Caspi, R., Fulcher, C.A., Latendresse, M., Kothari, A., Keseler, I.M., Krummenacker, M., Midford, P.E., Ong, Q., et al. (2019). The BioCyc collection of microbial genomes and metabolic pathways. Brief. Bioinformatics 20, 1085-1093.

757 Keller, K.L., Bender, K.S., and Wall, J.D. (2009). Development of a markerless genetic exchange system 758 for Desulfovibrio vulgaris Hildenborough and its use in generating a strain with increased transformation 759 efficiency. Appl. Environ. Microbiol. 75, 7682-7691.

Kip, N., and van Veen, J.A. (2015). The dual role of microbes in corrosion. ISME J. 9, 542-551. Symporter in Escherichia coli. J. Bacteriol. 200.

Kuehl, J.V., Price, M.N., Ray, J., Wetmore, K.M., Esquivel, Z., Kazakov, A.E., Nguyen, M., Kuehn, R., Davis, R.W., Hazen, T.C., et al. (2014). Functional genomics with a comprehensive library of transposon mutants for the sulfate-reducing bacterium Desulfovibrio alaskensis G20. MBio 5, e01041-14. in metabolic pathways of sulfate reduction in intestinal bacteria. Cells 9. 
Larsen, R.A., Wilson, M.M., Guss, A.M., and Metcalf, W.W. (2002). Genetic analysis of pigment

771 biosynthesis in Xanthobacter autotrophicus Py2 using a new, highly efficient transposon mutagenesis

772 system that is functional in a wide variety of bacteria. Arch. Microbiol. 178, 193-201.

773 Lin, S., and Cronan, J.E. (2011). Closing in on complete pathways of biotin biosynthesis. Mol. Biosyst. 7 , 774 1811-1821.

775 Mehta-Kolte, M.G., Stoeva, M.K., Mehra, A., Redford, S.A., Youngblut, M.D., Zane, G., Grégoire, P., 776 Carlson, H.K., Wall, J., and Coates, J.D. (2019). Adaptation of Desulfovibrio alaskensis G20 to perchlorate, 777 a specific inhibitor of sulfate reduction. Environ. Microbiol. 21, 1395-1406.

778 Meyer, B., Kuehl, J.V., Price, M.N., Ray, J., Deutschbauer, A.M., Arkin, A.P., and Stahl, D.A. (2014). The 779 energy-conserving electron transfer system used by Desulfovibrio alaskensis strain G20 during pyruvate 780 fermentation involves reduction of endogenously formed fumarate and cytoplasmic and membrane-bound 781 complexes, Hdr-Flox and Rnf. Environ. Microbiol. 16, 3463-3486.

782 Mikheenko, I.P., Rousset, M., Dementin, S., and Macaskie, L.E. (2008). Bioaccumulation of palladium by 783 Desulfovibrio fructosivorans wild-type and hydrogenase-deficient strains. Appl. Environ. Microbiol. 74, $784 \quad 6144-6146$.

785 Muyzer, G., and Stams, A.J.M. (2008). The ecology and biotechnology of sulphate-reducing bacteria. Nat. 786 Rev. Microbiol. 6, 441-454.

787 Novichkov, P.S., Kazakov, A.E., Ravcheev, D.A., Leyn, S.A., Kovaleva, G.Y., Sutormin, R.A., Kazanov, 788 M.D., Riehl, W., Arkin, A.P., Dubchak, I., et al. (2013). RegPrecise 3.0--a resource for genome-scale 789 exploration of transcriptional regulation in bacteria. BMC Genomics 14, 745.

790 van Opijnen, T., and Camilli, A. (2013). Transposon insertion sequencing: a new tool for systems-level 791 analysis of microorganisms. Nat. Rev. Microbiol. 11, 435-442.

792 van Opijnen, T., Bodi, K.L., and Camilli, A. (2009). Tn-seq: high-throughput parallel sequencing for fitness 793 and genetic interaction studies in microorganisms. Nat. Methods 6, 767-772.

794 Pan, S., Nikolakakis, K., Adamczyk, P.A., Pan, M., Ruby, E.G., and Reed, J.L. (2017). Model-enabled gene 795 search (MEGS) allows fast and direct discovery of enzymatic and transport gene functions in the marine 796 bacterium Vibrio fischeri. J. Biol. Chem. 292, 10250-10261.

797 Peck, H.D. (1962). The role of adenosine-5'-phosphosulfate in the reduction of sulfate to sulfite by 798 Desulfovibrio desulfuricans. J. Biol. Chem. 237, 198-203.

799 Price, M.N., and Arkin, A.P. (2017). PaperBLAST: Text Mining Papers for Information about Homologs. 
MSystems 2.

801 Price, M.N., Deutschbauer, A.M., Kuehl, J.V., Liu, H., Witkowska, H.E., and Arkin, A.P. (2011). Evidence-

802 based annotation of transcripts and proteins in the sulfate-reducing bacterium Desulfovibrio vulgaris

803 Hildenborough. J. Bacteriol. 193, 5716-5727.

804 Price, M.N., Ray, J., Wetmore, K.M., Kuehl, J.V., Bauer, S., Deutschbauer, A.M., and Arkin, A.P. (2014).

805 The genetic basis of energy conservation in the sulfate-reducing bacterium Desulfovibrio alaskensis G20.

806 Front. Microbiol. 5, 577.

807 Price, M.N., Wetmore, K.M., Waters, R.J., Callaghan, M., Ray, J., Liu, H., Kuehl, J.V., Melnyk, R.A., 808 Lamson, J.S., Suh, Y., et al. (2018). Mutant phenotypes for thousands of bacterial genes of unknown 809 function. Nature 557, 503-509.

810 Rajeev, L., Luning, E.G., Dehal, P.S., Price, M.N., Arkin, A.P., and Mukhopadhyay, A. (2011). Systematic 811 mapping of two component response regulators to gene targets in a model sulfate reducing bacterium.

812 Genome Biol. 12, R99.

813 Rajeev, L., Garber, M.E., Zane, G.M., Price, M.N., Dubchak, I., Wall, J.D., Novichkov, P.S., 814 Mukhopadhyay, A., and Kazakov, A.E. (2018). A new family of transcriptional regulators of 815 tungstoenzymes and molybdate/tungstate transport. Environ. Microbiol.

816 Rey, F.E., Gonzalez, M.D., Cheng, J., Wu, M., Ahern, P.P., and Gordon, J.I. (2013). Metabolic niche of a 817 prominent sulfate-reducing human gut bacterium. Proc Natl Acad Sci USA 110, 13582-13587.

818 Reznikoff, W.S. (2008). Transposon Tn5. Annu. Rev. Genet. 42, 269-286.

819 Riederer-Henderson, M.A., and Wilson, P.W. (1970). Nitrogen fixation by sulphate-reducing bacteria. J. 820 Gen. Microbiol. 61, 27-31.

821 Rodionov, D.A., Dubchak, I., Arkin, A., Alm, E., and Gelfand, M.S. (2004). Reconstruction of regulatory 822 and metabolic pathways in metal-reducing delta-proteobacteria. Genome Biol. 5, R90.

823 Rubin, B.E., Wetmore, K.M., Price, M.N., Diamond, S., Shultzaberger, R.K., Lowe, L.C., Curtin, G., Arkin, 824 A.P., Deutschbauer, A., and Golden, S.S. (2015). The essential gene set of a photosynthetic organism. Proc 825 Natl Acad Sci USA 112, E6634-43.

826 Rückert, C. (2016). Sulfate reduction in microorganisms-recent advances and biotechnological 827 applications. Curr. Opin. Microbiol. 33, 140-146.

828 Saier, M.H., Reddy, V.S., Tsu, B.V., Ahmed, M.S., Li, C., and Moreno-Hagelsieb, G. (2016). The 829 Transporter Classification Database (TCDB): recent advances. Nucleic Acids Res. 44, D372-9. 
Shatsky, M., Allen, S., Gold, B.L., Liu, N.L., Juba, T.R., Reveco, S.A., Elias, D.A., Prathapam, R., He, J., Yang, W., et al. (2016a). Bacterial interactomes: interacting protein partners share similar function and are validated in independent assays more frequently than previously reported. Mol. Cell. Proteomics 15, 15391555.

Shatsky, M., Dong, M., Liu, H., Yang, L.L., Choi, M., Singer, M.E., Geller, J.T., Fisher, S.J., Hall, S.C., Hazen, T.C., et al. (2016b). Quantitative Tagless Copurification: A Method to Validate and Identify ProteinProtein Interactions. Mol. Cell. Proteomics 15, 2186-2202.

Smart, J.P., Cliff, M.J., and Kelly, D.J. (2009). A role for tungsten in the biology of Campylobacter jejuni: tungstate stimulates formate dehydrogenase activity and is transported via an ultra-high affinity $\mathrm{ABC}$ system distinct from the molybdate transporter. Mol. Microbiol. 74, 742-757.

Smith, A.M., Heisler, L.E., Mellor, J., Kaper, F., Thompson, M.J., Chee, M., Roth, F.P., Giaever, G., and Nislow, C. (2009). Quantitative phenotyping via deep barcode sequencing. Genome Res. 19, 1836-1842.

Stoeva, M.K., and Coates, J.D. (2019). Specific inhibitors of respiratory sulfate reduction: towards a mechanistic understanding. Microbiology (Reading, Engl) 165, 254-269.

Thrasher, D.R., and Vance, I. (2005). Reservoir souring: mechanisms and prevention. In Petroleum Microbiology, B. Ollivier, and M. Magot, eds. (American Society of Microbiology), pp. 123-142.

Turkarslan, S., Raman, A.V., Thompson, A.W., Arens, C.E., Gillespie, M.A., von Netzer, F., Hillesland, K.L., Stolyar, S., López García de Lomana, A., Reiss, D.J., et al. (2017). Mechanism for microbial population collapse in a fluctuating resource environment. Mol. Syst. Biol. 13, 919.

Vita, N., Valette, O., Brasseur, G., Lignon, S., Denis, Y., Ansaldi, M., Dolla, A., and Pieulle, L. (2015). The primary pathway for lactate oxidation in Desulfovibrio vulgaris. Front. Microbiol. 6, 606.

Wetmore, K.M., Price, M.N., Waters, R.J., Lamson, J.S., He, J., Hoover, C.A., Blow, M.J., Bristow, J., Butland, G., Arkin, A.P., et al. (2015). Rapid quantification of mutant fitness in diverse bacteria by sequencing randomly bar-coded transposons. MBio 6, e00306-15.

Yong, P., Rowson, N.A., Farr, J.P.G., Harris, I.R., and Macaskie, L.E. (2002). Bioaccumulation of palladium by Desulfovibrio desulfuricans. J. Chem. Technol. Biotechnol. 77, 593-601.

857 gene encoding a hypothetical protein on sulfate reduction in Desulfovibrio vulgaris Hildenborough. Appl. 858 Environ. Microbiol. 76, 5500-5509.

859 Zhang, R., Ou, H.-Y., and Zhang, C.-T. (2004). DEG: a database of essential genes. Nucleic Acids Res. 32, 860 D271-2. 
bioRxiv preprint doi: https://doi.org/10.1101/2021.01.13.426591; this version posted January 13,2021 . The copyright holder for this preprint (which was not certified by peer review) is the author/funder, who has granted bioRxiv a license to display the preprint in perpetuity. It is made available under aCC-BY-ND 4.0 International license.

861 